\title{
Method for Solving Polynomial Equations
}

\section{Nahon YJ*}

Department of Mathematics, Technion-Israel Institute of Technology, Ashdod, Israel

\begin{abstract}
The purpose of my paper is to bring a method for solving polynomial equations using basic algebra and series and also using combinatorics. A series which converges to the solutions of polynomial equations. The contribution of this method is that it leads directly to precise results to find the roots of a polynomial equation of any degree starting from second degree to infinity and also for the solving of radicals since radicals are a particular type of polynomial equations for example to find the square root of 2 sends to solve the equation $x^{2}=2$. A general formula for the series which converges to the solutions of polynomial equations. For complex solutions we write for a polynomial $P(x)$, $\mathrm{P}(\mathrm{a}+\mathrm{bi})=\mathrm{P}(\mathrm{a}-\mathrm{bi})=0$ and to solve this separately for imaginary part and real part of the solution sends to solve for regular polynomial equations at one variable so we can use the method which is developed to find the solutions.
\end{abstract}

Keywords: Polynomial Equations; Coefficients; Series; Recurrent Relationships; Finite number

\section{Introduction}

\section{Nahon theorem}

If a $\mathrm{n} 0$ th polynomial equation in powers of $\mathrm{X}$ :

$\mathrm{X} \quad(\mathrm{n} 0)=\mathrm{A}(\mathrm{n} 0-1)^{\star} \mathrm{X}(\mathrm{n} 0-1)+\mathrm{A}(\mathrm{n} 0-2)^{*} \mathrm{X} \quad(\mathrm{n} 0-2)+\mathrm{A}(\mathrm{n} 0-3)^{*} \mathrm{X} \quad(\mathrm{n} 0-$ $3)+\ldots . . \mathrm{A}(1)^{\star} \mathrm{X}+\mathrm{A}(0)$ with the condition in the coefficients [1]

$\mathrm{A}(\mathrm{n} 0-1)^{\star} \mathrm{A}(0) \neq 0$

has a solution $\mathrm{X}$ in the set of real numbers $\mathrm{R}$ then if we define a series $\mathrm{E}(\mathrm{n})$ with $\mathrm{E}(\mathrm{n} 0)=\mathrm{A}(\mathrm{n} 0-1)$ and with the recurrent formula [2]:

$\mathrm{E}(\mathrm{n}+1)=\mathrm{A}(\mathrm{n} 0-1)^{\star} \mathrm{E}(\mathrm{n})+\mathrm{A}(\mathrm{n} 0-2)^{\star} \mathrm{E}(\mathrm{n}-1)+\mathrm{A}(\mathrm{n} 0-3)^{\star} \mathrm{E}(\mathrm{n}-2)+\ldots \ldots$ $\mathrm{A}(1)^{\star} \mathrm{E}(\mathrm{n}-\mathrm{n} 0+2)+\mathrm{A}(0)^{\star} \mathrm{E}(\mathrm{n}-\mathrm{n} 0+1)$ for $(\mathrm{n} \geq \mathrm{n} 0)$. Then

$$
L=\lim _{(n \rightarrow \infty)} \frac{E(n+1)}{E(n)} \text { is a solution of the } \mathrm{n} 0 \text { th polynomial equation }
$$

[3]. So $\mathrm{X}=\mathrm{L}$.

Condition of convergence [4]: for $\mathrm{i}=1,2, \ldots \ldots(\mathrm{n}-1)$

$A(i) \neq 0 \rightarrow\left[A(\mathrm{n} 0-1)^{\star} A(i)+A(i-1)\right] \neq 0$.

\section{Proof of the Nahon theorem}

$X^{(\mathrm{n} 0)}=A(n 0-1)^{\star} X^{(n 0-1)+} A(n 0-2)^{\star} X^{(n 0-2)}+A(n 0-3)^{\star} X^{(n 0-3)+} \ldots . A(1)^{\star} X+A(0)$

$X^{(n 0+1)}=X^{(\mathrm{n} 0) *} X$

Multiplication of the two sides by $\mathrm{X}$ leads to [5]:

$X^{(n 0+1)}=A(\mathrm{n} 0-1)^{\star} X^{(n 0)}+A(n 0-2)^{\star} X^{(\mathrm{n} 0-1)}+A(n 0-3) * \mathrm{X}^{(\mathrm{n} 0-2)}+\ldots$ $A(1)^{\star} X^{2}+A(0)^{\star} X$

Substituting $\mathrm{X}^{(\mathrm{n} 0)}$

by its expression in the second expression for $\mathrm{X}^{(\mathrm{n} 0+1)}$ leads to [6]:

$X^{(\mathrm{n} 0+1)}=A(n 0-1) \times\left[A(\mathrm{n} 0-1)^{\star} X^{(n 0-1)}+A(n 0-2)^{\star} X^{(n 0-2)}+A(n 0-3)^{\star} X^{(n 0-3)+} \ldots\right.$

$\left.A(1)^{\star} X+A(0)\right]+A(n 0-2)^{\star} X^{(n 0-1)+} A(n 0-3)^{\star} X^{(n 0-2)+} \ldots$.

$A(1)^{\star} X^{2}+A(0)^{\star} X=($ Grouping together the coefficients of the same powers)

$X^{(n 0+1)}=X^{(n 0-1)} \times\left[A(n 0-1)^{2}+A^{(n 0-2)}\right]+X^{(n 0-2)} \times\left[A(n 0-1)^{\star} A(n 0-\right.$ 2) $+A(n 0-3)]$
$\left.+X^{(n 0-3)} \times\left[A(n 0-1)^{*} A^{\prime} \imath 0-3\right)+A(n 0-4)\right]+\ldots X \times[A(n 0-$ $\left.1)^{\star} A(1)+A(0)\right]+A(n 0-1)^{\star} A(0)$

So in this manner by multiplication by $X$ and then substitution of $\mathrm{X}^{(\mathrm{n} 0)}$ by its expression we can express all the subsequent powers [7]

$$
X^{(n 0+2)}, X^{(n 0+3)}, X^{(n 0+4)}, \ldots \ldots, X^{(n 0+\mathrm{k})}
$$

in terms of a basis composed of the powers $\left[X^{(n 0-1)}, X^{(n 0-2)}, X^{(n 0-3)}, \ldots . X, X^{(0)}\right]$

So let's denote the coefficients of each power contained in this basis by series $E(n)$ [8],

$$
\mathrm{E}(2(\mathrm{n})), \mathrm{E}(3(\mathrm{n})), \ldots \ldots . . \mathrm{E}(\mathrm{n} 0-1(\mathrm{n})), \mathrm{E}(\mathrm{n} 0(\mathrm{n}))(\text { For } \mathrm{n} \geq \mathrm{n} 0)
$$

So we can write

$X^{(n 0)}=E(n 0)^{\star} X^{(n 0-1)}+E(2(n 0))^{\star} X^{(n 0-2)+} E(3(n 0))^{\star} X^{(n 0-3)+} \ldots E(n 0-1(n 0))^{\star} X$

$+E(n 0(n 0))$

Similarly we can write:

$X^{(n 0+1)}=E(n 0+1)^{\star} X^{(n 0-1)}+E(2(n 0+1))^{\star} X^{(n 0-2)}+E(3(n 0+1))^{\star} X^{(n 0-3)}+\ldots \ldots$ $E(n 0-1(n 0+1))^{\star} X+E(n 0(n 0+1))$

Since $X^{(n 0+1)}=X^{(n 0) \star} X$

So $X^{(n 0+1)=} E(n 0)^{\star} X \quad{ }^{(n 0)+} E(2(n 0))^{\star} X^{(n 0-1)}+E(3(n 0))^{\star} X^{(n 0-2)}+\ldots . E(n 0-$ $1(n 0))^{\star} X^{2}+E(n 0(n 0))^{\star} X$

Substitution of $X^{(n 0)}$ by its expression leads to:

$X^{(\mathrm{n} 0+1)=} E(n 0) \times\left[A(n 0-1)^{\star} X \quad(n 0-1)+A(n 0-2)^{\star} X \quad\left({ }^{(n 0-2)+} A(n 0-3)^{\star} X \quad\right.\right.$ $\left.{ }^{3)}+A(1)^{\star} X+A(0)\right]+E(2(n 0))^{\star} X^{(n 0-1)+} E(3(n 0))^{\star} X^{(n 0-2)}+\ldots . . E(4(n 0))^{\star} X^{(n 0-}$ ${ }^{3)+} E(n 0-1(n 0))^{\star} X^{2}+E(n 0(n 0))^{\star} X=($ Grouping together the coefficients of the same powers)

*Corresponding author: Julien Y, Department of Mathematics, TechnionIsrael Institute Of Technology, Ashdod, Israel, Tel: 00972-52-742-1339; E-mail: nahonyehoudajulien@yahoo.fr

Received August 21, 2017; Accepted August 04, 2018; Published August 07 2018

Citation: Julien Y (2018) Method for Solving Polynomial Equations. J Appl Computat Math 7: 409. doi: 10.4172/2168-9679.1000409

Copyright: @ 2018 Julien Y. This is an open-access article distributed under the terms of the Creative Commons Attribution License, which permits unrestricted use, distribution, and reproduction in any medium, provided the original author and source are credited. 
$X^{(n 0+1)}=X \quad{ }^{(n 0-1)} \times\left[A(n 0-1)^{\star} E(n 0)+E(2(n 0))\right]+X \quad{ }^{(n 0-2)} \times[A(n 0-$ $\left.2)^{\star} E(n 0)+E(3(n 0))\right]+X^{(n 0-3)} \times\left[A(n 0-3)^{\star} E(n 0)+E(4(n 0))\right]+\ldots \ldots .$. $\mathrm{X}^{2} \times\left[\mathrm{A}(2)^{\star} \mathrm{E}(\mathrm{n} 0)+\mathrm{A}(1)\right]+\mathrm{X} \times\left[\mathrm{A}(1)^{\star} \mathrm{E}(\mathrm{n} 0)+\mathrm{E}(\mathrm{n} 0(\mathrm{n} 0))\right]+\mathrm{A}(0)^{\star} \mathrm{E}(\mathrm{n} 0)$

So we can relate the coeficientes

$\mathrm{E}(\mathrm{n} 0+1)=\mathrm{A}(\mathrm{n} 0-1)^{\star} \mathrm{E}(\mathrm{n} 0)+\mathrm{E}(2(\mathrm{n} 0))=\mathrm{A}(\mathrm{n} 0-1)^{2}+\mathrm{A}(\mathrm{n} 0-2)$

$\mathrm{E}(2(\mathrm{n} 0+1))=\mathrm{A}(\mathrm{n} 0-2)^{*} \mathrm{E}(\mathrm{n} 0)+\mathrm{E}(3(\mathrm{n} 0))=\mathrm{A}(\mathrm{n} 0-1) * \mathrm{~A}(\mathrm{n} 0-2)+\mathrm{A}(\mathrm{n} 0-3)$

$\mathrm{E}(3(\mathrm{n} 0+1))=\mathrm{A}(\mathrm{n} 0-3)^{*} \mathrm{E}(\mathrm{n} 0)+\mathrm{E}(4(\mathrm{n} 0))=\mathrm{A}(\mathrm{n} 0-1)^{*} \mathrm{~A}(\mathrm{n} 0-3)+\mathrm{A}(\mathrm{n} 0-4)$

$\mathrm{E}(\mathrm{n} 0-2(\mathrm{n} 0+1))=\mathrm{A}(2)^{\star} \mathrm{E}(\mathrm{n} 0)+\mathrm{E}(\mathrm{n} 0-1(\mathrm{n} 0))=\mathrm{A}(\mathrm{n} 0-1)^{\star} \mathrm{A}(2)+\mathrm{A}(1)$

$\mathrm{E}(\mathrm{n} 0-1(\mathrm{n} 0+1))=\mathrm{A}(1) \star \mathrm{E}(\mathrm{n} 0)+\mathrm{E}(\mathrm{n} 0(\mathrm{n} 0))=\mathrm{A}(\mathrm{n} 0-1)^{\star} \mathrm{A}(1)+\mathrm{A}(0)$

$\mathrm{E}(\mathrm{n} 0(\mathrm{n} 0+1))=\mathrm{A}(0)^{\star} \mathrm{E}(\mathrm{n} 0)=\mathrm{A}(0)^{\star} \mathrm{A}(\mathrm{n} 0-1)$

From these recurrent relationships following the pattern of recurrence we can write:

$\mathrm{E}(\mathrm{n} 0+1)=\mathrm{A}(\mathrm{n} 0-1) * \mathrm{E}(\mathrm{n} 0)+\mathrm{E}(2(\mathrm{n} 0))=\mathrm{A}(\mathrm{n} 0-1) * \mathrm{E}(\mathrm{n} 0)+\mathrm{A}(\mathrm{n} 0-$ $2)^{\star} \mathrm{E}(\mathrm{n} 0-1)+\mathrm{E}(3(\mathrm{n} 0-1))=$

$\mathrm{A}(\mathrm{n} 0-1)^{\star} \mathrm{E}(\mathrm{n} 0)+\mathrm{A}(\mathrm{n} 0-2) * \mathrm{E}(\mathrm{n} 0-1)+\mathrm{A}(\mathrm{n} 0-3) * \mathrm{E}(\mathrm{n} 0-2)+\mathrm{E}(4(\mathrm{n} 0-2))=$

$\mathrm{A}(\mathrm{n} 0-1){ }^{\star} \mathrm{E}(\mathrm{n} 0)+\mathrm{A}(\mathrm{n} 0-2)^{\star} \mathrm{E}(\mathrm{n} 0-1)+\mathrm{A}(\mathrm{n} 0-3) * \mathrm{E}(\mathrm{n} 0-2)+\mathrm{A}(\mathrm{n} 0-$ $4)^{\star} \mathrm{E}(\mathrm{n} 0-3)+\ldots \mathrm{A}(1)^{\star} \mathrm{E}(2)+\mathrm{A}(0)^{\star} \mathrm{E}$

To write this the following condition is needed: $\mathrm{E}(\mathrm{n} 0+1) \neq 0$ and $E(i(n 0+1) \neq 0$ for $i=2,3, \ldots . n 0$. so we can write the condition as follows:

\section{$\left[\mathrm{A}(\mathrm{n} 0-1)^{\star} \mathrm{A}(\mathrm{i})+\mathrm{A}(\mathrm{i}-1) \neq 0\right]$}

if this condition applies so it is following from the recurrent relationships $\mathrm{E}(\mathrm{i}(\mathrm{n}))$ that for $\mathrm{n} \geq \mathrm{n} 0 E(n) \neq 0$ and therefore the condition of convergence of the ratio

$\frac{E(n+1)}{E(n)}$ in infinity is satisfied.

So from this we can write a recurrent relationship for $\mathrm{E}(\mathrm{n})$ :

$\mathrm{E}(\mathrm{n}+1)=\mathrm{A}(\mathrm{n} 0-1)^{\star} \mathrm{E}(\mathrm{n})+\mathrm{A}(\mathrm{n} 0-2)^{\star} \mathrm{E}(\mathrm{n}-1)+\mathrm{A}(\mathrm{n} 0-3)^{\star} \mathrm{E}(\mathrm{n}-2)+\ldots \ldots \ldots$ $. \mathrm{A}(1)^{\star} \mathrm{E}(\mathrm{n}-\mathrm{n} 0+2)+\mathrm{A}(0)^{*} \mathrm{E}(\mathrm{n}-\mathrm{n} 0+1)$

Let's define $L=\lim _{n \rightarrow \infty}=\frac{E(n+1)}{E(n)}$

$\mathrm{E}(\mathrm{n}+1) / \mathrm{E}(\mathrm{n})=\mathrm{A}(\mathrm{n} 0-1)+\mathrm{A}(\mathrm{n} 0-2) \times[E(n-1) / E(n)]+A(n 0-3) \times[E(n-$ $2) / E(n)]+\ldots$

$A(1) \times[E(n-n 0+2) / E(n)]+A(0) \times[E(n-n 0+1) / E(n)]$

Let's define $G(n+1)=E(n+1) / E(n)$ so we can write:

$\mathrm{G}(\mathrm{n}+1)=\mathrm{A}(\mathrm{n} 0-1)+\mathrm{A}(\mathrm{n} 0-2) / \mathrm{Gn}+\mathrm{A}(\mathrm{n} 0-3) /\left[\mathrm{Gn}{ }^{\star} \mathrm{G}(\mathrm{n}-1)\right]+\ldots \ldots \ldots$ $\ldots .+A(1) /\left[G(n)^{\star} G(n-1)^{\star} \ldots G(n-n 0+3)\right]+A(0) /\left[G n^{\star} G(n-1)^{\star} \ldots \ldots\right.$ $\mathrm{G}(\mathrm{n}-\mathrm{n} 0+2)]$

$$
\begin{aligned}
& L=\lim _{n \rightarrow \infty}=\frac{E(n+1)}{E(n)}=\lim _{n \rightarrow \infty} G(n+1)= \\
& \lim _{n \rightarrow \infty} G(n)=\lim _{n \rightarrow \infty} G(n-1)= \\
& \lim _{n \rightarrow \infty} G(n-n 0+2) \\
& \text { So } \mathrm{L}=\mathrm{A}(\mathrm{n} 0-1)+\mathrm{A}(\mathrm{n} 0-2) / \mathrm{L}+\mathrm{A}(\mathrm{n} 0-3) / \mathrm{L}^{2}+\ldots . . \mathrm{A}(1) / \mathrm{L}^{(\mathrm{n} 0-2)+} \mathrm{A}(0) / \mathrm{L}^{(\mathrm{n} 0-1)}
\end{aligned}
$$

$\mathrm{L}^{(\mathrm{n} 0)}=A(n 0-1)^{\star} L^{(n 0-1)}+A(n 0-2)^{\star} L^{(n 0-2)+} A(n 0-3)^{\star} L^{(n 0-3)+} \ldots \ldots$. $A(1)^{\star} L+A(0)$
So $\mathrm{X}=\mathrm{L}$ is a solution of the n0th polynomial equation

$\mathrm{X}^{(n 0)}=A(n 0-1)^{\star} X^{(n 0-1)}+A(n 0-2)^{\star} X^{(n 0-2)}+A(n 0-3)^{\star} X^{(n 0-3)} \ldots \ldots . A(1)^{\star} X+A(0)$.

\section{Particular cases:}

* When $\mathrm{A}(\mathrm{n} 0-1)=0$

Before being able to solve the equation, we first need to transform the equation into a classical n0 order equation when at least the coefficient of $\mathrm{x}^{\mathrm{n} 0-1}$ and $\mathrm{x}^{0}$ will be different from 0 . This can be done by setting $\mathrm{X}=\mathrm{x}+\mathrm{a}$, then we can solve the new equation in $\mathrm{x}$.

Example: Let's get a look at the equation $\mathrm{X}^{3}=3 \mathrm{X}+4$

Substitution of $\mathrm{X}$ by $\mathrm{x}+\mathrm{a}$ where " $\mathrm{a}$ " can be any real leads to:

$(x+a)^{3}=3(x+a)+4$

so developing the cubic form leads to:

$x^{3}+a^{3}+3 a^{2} x+3 a x^{2}=3 x+3 a+4$ this leads to:

$\mathrm{x}^{3}=-3 \mathrm{a} x^{2}+x\left(3-3 \mathrm{a}^{2}\right)+(3 \mathrm{a}+4)-\mathrm{a}^{3}$

So $\mathrm{X}=\mathrm{x}+\mathrm{a}$ is the solution of the cubic equation $\mathrm{X}^{3}=3 \mathrm{X}+4$

${ }^{\star}$ If for a particular $\mathrm{Ai} \neq 0 A(n 0-1)^{\star} A(i)+A(i-1)=0$

We need to transform the equation for $\mathrm{i}$ by setting $\mathrm{X}=\mathrm{x}+\mathrm{a}$ and then we need to recalculate the next terms of the series $\mathrm{E}(\mathrm{k})$.

\section{Complex Solutions}

For a polynomial $\mathrm{P}(\mathrm{X})=0, \mathrm{P}(\mathrm{a}+\mathrm{bi})=\mathrm{P}(\mathrm{abi})=0$ so we need to solve separately for the real parts and imaginary parts so it sends to solve a regular polynomial equation at one variable to find $\mathrm{a}$ and $\mathrm{b}$

\section{Example}

Let's try to solve a seventic $(\mathrm{n} 0=7)$ equation

$X^{7}=2 X^{6}+4 X^{5}+3 X^{4}+2 X^{3}+X^{2}+X+6$

Let's express the recurrent relationship for $\mathrm{E}(\mathrm{n})$ and $\mathrm{G}(\mathrm{n})$ :

$\mathrm{E}(\mathrm{n}+1)=2 \mathrm{E}(\mathrm{n})+4 \mathrm{E}(\mathrm{n}-1)+3 \mathrm{E}(\mathrm{n}-2)+2 \mathrm{E}(\mathrm{n}-3)+\mathrm{E}(\mathrm{n}-4)+\mathrm{E}(\mathrm{n}-$ $5)+6 E(n-6)$

$\mathrm{G}(\mathrm{n}+1)=\mathrm{E}(\mathrm{n}+1) / \mathrm{E}(\mathrm{n})$

$\mathrm{G}(\mathrm{n}+1)=2+4 / \mathrm{G}(\mathrm{n})+3 /\left[\mathrm{G}(\mathrm{n})^{\star} \mathrm{G}(\mathrm{n}-1)\right]+2 /\left[\mathrm{G}(\mathrm{n})^{\star} \mathrm{G}(\mathrm{n}-1)^{\star} \mathrm{G}(\mathrm{n}-2)\right]+$

$1 /\left[G(n){ }^{\star} G(n-1)^{\star} G(n-2)^{\star} G(n-3)\right]+1 /\left[G(n) * G(n-1)^{\star} G(n-2)^{\star} G(n-\right.$ $\left.3)^{*} \mathrm{G}(\mathrm{n}-4)\right]+$

\section{$6 /\left[G(n)^{\star} G(n-1)^{\star} G(n-2)^{\star} G(n-3)^{\star} G(n-4)^{\star} G(n-5)\right]$}

Before we can use those formulas we need to calculate the first six terms for $\mathrm{E}(\mathrm{n})$ and

$G(n)$ :

$\mathrm{E}(8), \mathrm{E}(9), \mathrm{E}(10), \mathrm{E}(11), \mathrm{E}(12), \mathrm{E}(13)$

$\mathrm{G}(8), \mathrm{G}(9), \mathrm{G}(10), \mathrm{G}(11), \mathrm{G}(12), \mathrm{G}(13)$

$X^{8}=X^{7} X=2\left[2 X^{6}+4 X^{5}+3 X^{4}+2 X^{3}+X^{2}+X+6\right]+$

$4 X^{6}+3 X^{5}+2 X^{4}+X^{3}+X^{2}+6 X=8 X^{6}+11 X^{5}+8 X^{4}+5 X^{3}+3 X^{2}+8 X+12$

So $\mathrm{E}(8)=8$, from the seventic equation $\mathrm{E}(7)=2$ 


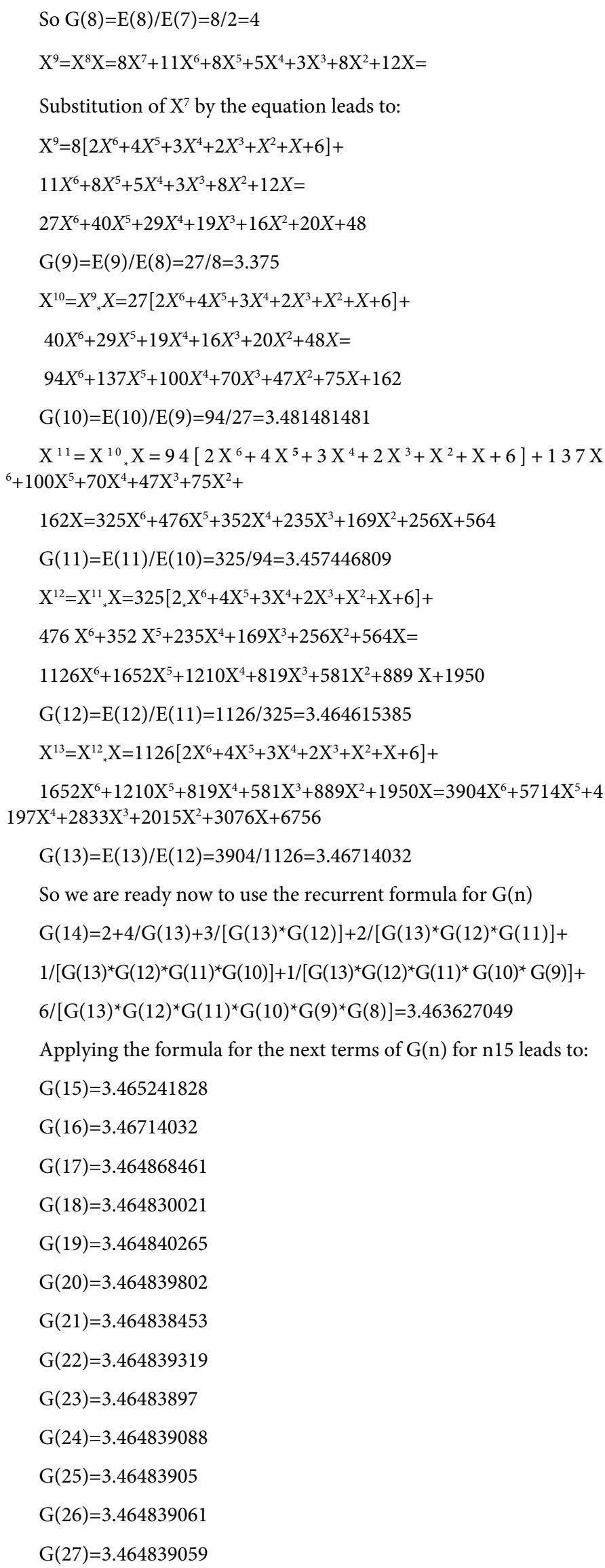

$$
\begin{aligned}
& G(28)=3.464839058 \\
& G(29)=3.464839059 \\
& G(30)=3.464839059
\end{aligned}
$$

Since $G(29)=G(30)=3.464839059$

So we can conclude that all the next terms of the series for $\mathrm{n} \geq 30$ will be equal to $G(30)$

So we can conclude that $L=\lim _{n \rightarrow \infty} G(n)=3.464839059$ checking this result will show that

$\mathrm{X}=\mathrm{L}=3.464839059$ is a solution of the seventic equation.

In this example, we saw that in fact (considering a finite number of digits after the decimal point) a finite number of iterations is needed to calculate the limit L.

So let's formulate a general result for the number of iterations needed to calculate the limit $\mathrm{L}$.

Let it be a no th polynomial equation:

$\mathrm{X}^{\mathrm{n} 0}=A(n 0-1)^{\star} X^{\mathrm{n} 0-1}+A(n 0-2)^{\star} X^{n 0-2}+A(n 0-3)^{\star} X^{n 0-3}+\ldots . A(1)^{\star} X+A(0)$

Let it be the coefficients[A(n0-1), A(n0-2), A(n0-3),A(n0-4),.. .. $\mathrm{A}(0)]$ real integers different from zero $[\mathrm{A}(\mathrm{i})$ integers $\mathrm{A}(\mathrm{i}) \neq 0$ and $\mathrm{A}(i) \in \mathfrak{R}]$

The number of iterations needed is determined by the minimum common factor Ko.

Ko is the minimum common factor between

$1 \times|A(n 0-1)|, 2 \times|A(n 0-2)|, 3 \times|A(n 0-3)|$ $(n 0-$ $1) \times|A(1)|, n 0 \times|A(0)|$.

Ko is determined by simplifying

$1 \times|A(n 0-1)|, \quad 2 \times|A(n 0-2)|, 3 \times|A(n 0-3)|, \ldots \ldots . .(n 0-1) \times|A(1)|$, $n 0 \times|A(0)|$

by each pair of prime factors (p,p) contained in the $\mathrm{i} \times|A(n 0-i)|$ for $i=1,2,3, \ldots \ldots .(n 0-1)$

and then by taking the product between the different prime factors in the $\mathrm{i} \times|A(n 0-i)|$,

Then we determine the equivalent of the product in modulo $\mathrm{n} 0$ the result is $\mathrm{k} 0[\mathrm{n} 0]$,

So ko+n0 iterations will always give at least one digit of the root solution X.

Then for $\mathrm{m}$ digits the number of iterations that will always give the $\mathrm{m}$ digits is

$\mathrm{K}=(\mathrm{Ko}+\mathrm{n} 0) \times[1+2+\ldots . . m]=(\mathrm{Ko}+n 0) \times\left[m^{\star}(m+1) / 2\right]$.

In the decimal system $\mathrm{K}+10$ iterations will be the maximum number of iterations needed that the $\mathrm{m}$ digits are also common to the subsequent terms of the serie $G(n)$.

This says that for $\mathrm{k} \geq \mathrm{K}+10$

$k$ iterations correspond to the indice $k+n 0$ for the serie $G(n)$

So $\mathrm{G}(\mathrm{k}+\mathrm{n} 0)$ contains the $\mathrm{m}$ digits of the root solution.

So $\mathrm{X}($ with $\mathrm{m}$ digits $)=$

$\operatorname{Int}\left[\mathrm{G}\left[(\mathrm{Ko}+\mathrm{n} 0)^{*} \mathrm{~m}^{*}(\mathrm{~m}+1) / 2+\mathrm{n} 0+10\right] \times 10^{(\mathrm{m})}\right] / 10^{(\mathrm{m})}$ 
If we call $\mathrm{k} 1=\mathrm{Ko}+\mathrm{n} 0$ and Int the integer value of a parameter we can express a general formula for the solution of polynomial equations:

$\mathrm{X}($ with $\mathrm{m}$ digits $)=\operatorname{Int}\left[\mathrm{G}\left[\mathrm{k} 1^{\star} \mathrm{m}^{\star}(\mathrm{m}+1) / 2+10\right] \times 10^{(\mathrm{m})}\right] / 10^{(\mathrm{m})}$

For calculating Ko, a rule is that only the $|\mathrm{A}(\mathrm{i})| \neq 0$ are taken into account for the product. In any case the minimum condition is

$$
|A(n 0-1)| \times|A(0)| \neq 0 \text {. }
$$

A particular case:

When $[\mathrm{A}(\mathrm{n} 0-1), \mathrm{A}(\mathrm{n} 0-2), \mathrm{A}(\mathrm{n} 0-3), \ldots \ldots \ldots \ldots \mathrm{A}(0)]$ are not integers we express their rational form $A(i)=A^{\prime}(i) / A^{\prime}(n 0)$

So we can express the equation in this form

$\mathrm{A}^{\prime}(\mathrm{n} 0)^{\star} \mathrm{X}^{(n)}=A^{\prime}(n 0-1)^{\star} X^{(n 0-1)+} A^{\prime}(n 0-2)^{\star} X^{(n 0-2)}+\ldots . . A^{\prime}(1)^{\star} X+A^{\prime}(0)$

$\left[A^{\prime}(n 0), A^{\prime}(n 0-1), A^{\prime}(\mathrm{n} 0-2), \ldots \ldots \ldots \mathrm{A}^{\prime}(0)\right] \neq 0$ and also real integers.

Then we can express the product for Ko

$$
\begin{aligned}
& K o=1 \times A^{\prime}(n 0-1) \times 2\left|A^{\prime}(n 0-2)\right| \times 3 \mid A^{\prime}(n 0-3) \times \ldots \ldots \\
& (n 0-1) \times A^{\prime}(1) \times(n 0) \times\left|A^{\prime}(0)\right|=n 0 !^{*} \prod_{i=1}^{i=n 0} *\left|A^{\prime}(n 0-i)\right|
\end{aligned}
$$

A rule is that only the $\left|A^{\prime}(i)\right| \neq 0$ are taken into account for the product. In any case the minimum condition is

$$
\left|A^{\prime}(n 0-1)\right| *\left|A^{\prime}(0)\right| \neq 0 \text {. }
$$

Now the question which can be raised up is how to know the number of digits which a rational solution may have.

Determination of the maximum number of digits for a rational solution in the decimal base

Let it be a $\mathrm{n} 0$ th polynomial equation

$\mathrm{X}^{(n 0)}=A(n 0-1) X^{(n 0-1)+} A(n 0-2) X^{(n 0-2)+} A(n 0-3) X^{(n 0-3)}+\ldots . . A(1)^{\star} X+A(0)$.

If $\mathrm{X}$ has $\mathrm{m}$ digits in the decimal base so the number of digits of the left side can be expressed $A m+B$ and in the right side can be expressed $\mathrm{Cm}+\mathrm{D}$, So since the left side is equal to the right side so $\mathrm{Am}+\mathrm{B}=\mathrm{Cm}+\mathrm{D}$

$(A-C)^{\star} m=D-B$ so $m=(D-B) /(A-C)$ The biggest value for $m$ can be obtained for $\mathrm{D}$ maximum, $\mathrm{B}$ minimum, $\mathrm{A}$ minimum, $\mathrm{C}$ maximum $\mathrm{C}$ maximum $=\mathrm{n} 0-1 \mathrm{~A}$ minimum $=\mathrm{n} 0 \mathrm{~B}$ minimum $=-\mathrm{n} 0+1$

$$
\begin{aligned}
& D=\left[\sum_{k=0}^{k=n 0-1} M(k)\right]+\sum_{k=1}^{k=n 0-1}[k-1] \\
& D=\left[\sum_{k=0}^{k=n 0-1} M(k)\right]+[(n 0-2) *(n 0-1)]
\end{aligned}
$$

So $\mathrm{m}=[\mathrm{D}+(\mathrm{n} 0-1)] /[\mathrm{n} 0-(\mathrm{n} 0-1)]=\mathrm{D}+\mathrm{n} 0-1$

So $\mathrm{m} \leq \mathrm{D}+n 0-1$

So $\mathrm{m}(\mathrm{Max})=\mathrm{D}+\mathrm{n} 0-1$

$$
m(\operatorname{Max})=\left[\sum_{k=0}^{k=n 0-1} M(k)\right]+[(n 0-2) *(n 0-1) / 2]+(n 0-1) .
$$

When $\mathrm{M}(\mathrm{k})$ is the number of digits for $\mathrm{A}(\mathrm{k})$ : example if $\mathrm{A}(\mathrm{n} 0$ $1)=13$ so $M(n 0-1)=2$.

What Henrik Niels Abel has demonstrated is that a general formula for the solution of polynomial equations can not be obtained in terms of radicals for degree $n \geq 5$.
However the equation $\mathrm{X}^{2}=2$ is in itself a mathematical problem to solve because saying that the solution of this equation is $X=\sqrt{2}$ does not give the arithmetic expression of the solution

In order to solve the equation $\mathrm{X}^{2}=2$ we need first to transform this equation into a classical second order equation. This can be done by transformation with $\mathrm{X}=\mathrm{x}+\mathrm{a}$

$(\mathrm{x}+\mathrm{a})^{2}=2$ this leads to:

$x^{2}+a^{2}+2 a x=2 \Leftrightarrow x^{2}=-2 a x+2-a^{2}$

One example that will lead to the solution can be $a=1$ this leads to $\mathrm{x}^{2}=-2 \mathrm{x}+2-1=-2 \mathrm{x}+1$

so we need to solve the equation.

$x^{2}=-2 x+1$ then $X=x+1$ corresponds to the solution of $X^{2}=2$ or in other terms we can express the square root of $2: \sqrt{2}=x+1$.

Let's solve the equation

$x^{2}=-2 x+1$

Let's express the recurrent relationship for $E(n)$ and $G(n)$

$\mathrm{E}(\mathrm{n}+1)=-2 \mathrm{E}(\mathrm{n})+\mathrm{E}(\mathrm{n}-1)$

$\mathrm{G}(\mathrm{n}+1)=-2+[1 / \mathrm{G}(\mathrm{n})]$

Before we can use those formulas we need first to calculate the first terms for $\mathrm{E}(\mathrm{n})$ and

$\mathrm{G}(\mathrm{n}): \mathrm{E}(3), \mathrm{G}$

$x^{3}=x^{2 *} x=-2 x^{2}+x=-2[-2 x+1]+x=4 x-2+x=5 x-2$

So $E(3)=5, G(3)=E(3) / E(2)=5 /(-2)=-2.5$

Applying the formula for $\mathrm{G}(\mathrm{n})$ leads to:

$\mathrm{G}(4)=-2+[1 /(-5 / 2)]=-2+(-2 / 5)=-2-0.4=-2.4$

$\mathrm{G}(4)=-2.4$

$G(5)=-2.416666667$

$G(6)=-2.413793103$

$G(7)=-2.414285714$

$G(8)=-2.414201183$

$G(9)=-2.414215686$

$\mathrm{G}(10)=-2.414213198$

$G(11)=-2.414213625$

$\mathrm{G}(12)=-2.414213552$

$G(13)=-2.414213564$

$\mathrm{G}(14)=-2.414213562$

$G(15)=-2.414213562$

Since $G(15)=G(14)=-2.414213562$.

So we can conclude that $\mathrm{x} 1=-2.414213562$ we can now find easily the other solution

$12-1(-2.414213562)$ 


\section{$1(-2.414213562+2) 0$}

The other solution is $\mathrm{x}^{2}=2.414213562-2=0.414213562$

$\mathrm{X} 1=\mathrm{x}+1=-2.414213562+1=-1.414213562$

$\mathrm{X} 2=0.414213562+1=1.414213562$

So those are the two square roots of 2

$-\sqrt{2}=-1.414213562$

$\sqrt{2}=1.414213562$.

So my conclusion is that since we can find the radicals in terms of simple operations like additions multiplications subtractions divisions so we should be able to find general formulas which can be expressed in terms of simple operations to express the solutions of any nth polynomial equation. All what is needed for this is to find explicit formulas for the series $E(n)$ and $G(n)$.

Let's try to develop a formula for the second degree polynomial equations. $\mathrm{X}^{2}=P X+Q$

let's express the recurrent relationship for $\mathrm{E}(\mathrm{n})$ :

$\mathrm{E}(\mathrm{n}+1)=\mathrm{P}^{\star} \mathrm{E}(\mathrm{n})+\mathrm{Q}^{\star} \mathrm{E}(\mathrm{n}-1)$

This is the formula for $\mathrm{E}(\mathrm{k})$ :when $\mathrm{k}=0$ corresponds to $\mathrm{n}=\mathrm{n} 0=2$ $(\mathrm{k}=\mathrm{n}-\mathrm{n} 0=\mathrm{n}-2)$

$\mathrm{E}(\mathrm{k})=\mathrm{P}^{(\mathrm{k}+1)}+\mathrm{k}\left[\mathrm{P}^{(\mathrm{k}-1)}\right] \times \mathrm{Q}+[(\mathrm{k}-2)(\mathrm{k}-1) / 2] \times\left[\mathrm{P}^{(\mathrm{k}-3)}\right] \times \mathrm{Q}^{2}+$

$[(\mathrm{k}-2)(\mathrm{k}-3)(\mathrm{k}-4) / 6] \times\left[\mathrm{P}^{(\mathrm{k}-5)}\right] \times \mathrm{Q}^{3}+[(\mathrm{k}-3)(\mathrm{k}-4)(\mathrm{k}-5)(\mathrm{k}-6) / 24] \times\left[\mathrm{P}^{(\mathrm{k}-7)}\right] \times \mathrm{Q}^{4}$

$+[(\mathrm{k}-4)(\mathrm{k}-5)(\mathrm{k}-6)(\mathrm{k}-7)(\mathrm{k}-8) / 120] \times\left[\mathrm{P}^{(\mathrm{k}-9)}\right] \times \mathrm{Q}^{5}+\ldots$

The final development of the formula leads to:

$$
\begin{aligned}
& E(k)=\left(\begin{array}{c}
k+1 \\
0
\end{array}\right) * P^{(k+1)}+\left(\begin{array}{c}
k \\
1
\end{array}\right) * P^{(k-1)} * Q+ \\
& \left(\begin{array}{c}
k-1 \\
2
\end{array}\right) * p^{(k-3)} * Q^{2}+\left(\begin{array}{c}
k-2 \\
3
\end{array}\right) * P^{(k-5)} * \mathrm{Q}^{3}+ \\
& \left(\begin{array}{c}
k-3 \\
4
\end{array}\right) * P^{(k-7)} * Q^{4}+\left(\begin{array}{c}
k-4 \\
5
\end{array}\right) * P^{(k-9)} * Q^{5}+\ldots .
\end{aligned}
$$

$\left(\begin{array}{l}L \\ L\end{array}\right) * Q^{L}$ (if $k$ is odd $) o r$

$$
\left.\left(\begin{array}{l}
L+1 \\
L
\end{array}\right) * P^{*} Q^{L} \text { (If } k \text { is even }\right) .
$$

The last term of the formula will depend on whether $\mathrm{k}$ is odd or $\mathrm{k}$ is even

Now we will try to obtain a general formula valid for any $\mathrm{n} 0$ th degree polynomial equation with coefficients $\mathrm{A}(\mathrm{n} 0-1), \mathrm{A}(\mathrm{n} 0-2), \mathrm{A}(\mathrm{n} 0$ 3),.......A(1),A(0) :

$\mathrm{X}^{(n 0)}=A(n 0-1)^{\star} X^{(n 0-1)}+A(n 0-2)^{\star} X^{(n 0-2)}+A(n 0-3)^{\star} X^{(n 0-3)}+\ldots \ldots$ $. A(1)^{\star} X+A(0)$

Let's express the recurrent relationship for $\mathrm{E}(\mathrm{k})$ :

$\mathrm{E}(\mathrm{k}+1)=\mathrm{A}(\mathrm{n} 0-1)^{\star} \mathrm{E}(\mathrm{k})+\mathrm{A}(\mathrm{n} 0-2)^{\star} \mathrm{E}(\mathrm{k}-1)+\mathrm{A}(\mathrm{n} 0-3)^{\star} \mathrm{E}(\mathrm{k}-2)+\ldots \ldots \ldots$ $\mathrm{A}(1)^{\star} \mathrm{E}(\mathrm{k}-(\mathrm{n} 0-2))+\mathrm{A}(0)^{\star} \mathrm{E}(\mathrm{k}-(\mathrm{n} 0-1))$
Developing a formula for $\mathrm{E}(\mathrm{k})$ leads to:

$$
\begin{aligned}
& E(k)=A(n 0-1)^{(k+1)}+ \\
& \left(\begin{array}{c}
k \\
1
\end{array}\right) * A(n 0-1)^{(k-1)} * A(n 0-2)+ \\
& \left(\begin{array}{c}
k-1 \\
1
\end{array}\right) * A(n 0-1)^{(k-2)} * A(n 0-3)+ \\
& \left(\begin{array}{c}
k-2 \\
1
\end{array}\right) * A(n 0-1)^{(k-3)} * A(n 0-4)+\ldots . . \\
& \left(\begin{array}{c}
k-n o+3 \\
1
\end{array}\right) * A(n 0-1)^{(k-n 0+2)} * A(1)+ \\
& \left(\begin{array}{c}
k-n 0+2 \\
2
\end{array}\right) * \mathrm{~A}(n o-1)^{(k-n 0+1)} * A(0)+ \\
& \left.\sum_{S=2}^{S=k}\left(\begin{array}{c}
S+K(n 0-1) \\
S
\end{array}\right) *\left[\prod_{i=n 0-1}^{i=n 0-2} A(i)^{K(i)}\right]\right] \\
& {\left[\prod_{i=0}^{i=0} A(i)^{K(i)}\right] \text { where } S \text { is defined to be }} \\
& S=\sum_{i=0}^{i=n 0-2} K(i)
\end{aligned}
$$

$\mathrm{K}(\mathrm{n} 0-1)$ is defined to be

$K(n 0-1)=k-\left[\sum_{i=0}^{i=n 0-2}(K(i) *(n 0-i))\right]+1$

with one condition: $\mathrm{K}(\mathrm{n} 0-1) \geq 0$

and $\mathrm{F}$ is a function defined as this:

$$
\begin{aligned}
& F\left(\left[A^{K}\right]=\frac{K !}{K !}=1 \quad \text { with } k \geq 0\right) \\
& F\left[\prod_{i=0}^{i=n 0-2} A(i)^{k(i)}\right]=\frac{\left.\left(\sum_{i=0}^{i=n 0-2} K(i)\right) !\right)}{\prod_{i=0}^{i=n 0-2}(K(i) !)}= \\
& \frac{S !}{i=n 0-2}(K(i) !) \\
& \prod_{i=0}^{i=n 0-2} K\left(i \text { th } S=\sum_{i=0}^{i} K(i)\right.
\end{aligned}
$$

In the formula for the serie $\mathrm{E}(\mathrm{k})$

\section{Example}

$$
F\left(A^{2} * B^{3} * C^{4}\right)=\frac{9 !}{2 ! * 3 ! * 4 !}=1260
$$

We can rewrite the formula for $\mathrm{E}(\mathrm{k})$ :

$$
\begin{aligned}
& E(k)=A(n 0-1)^{(k+1)}+ \\
& \sum_{i=0}^{i=n 0-2}\left(\begin{array}{c}
(k-(n-i-2)) \\
1
\end{array}\right) * A(n 0-1)^{(k-(n 0-i-1))} * A(i) \\
& +\sum_{S=2}^{S=k}\left(\left(\begin{array}{c}
\left(\sum_{i=0}^{i=n 0-2} K(i)+K(n 0-1)\right) \\
\left(\sum_{i=0}^{i=n 0-2} K(i)\right)
\end{array}\right) *\right. \\
& \left.\sum_{i=0}^{i=n 0-2} K(i)\right) ! \\
& \prod_{i=0}^{i=n 0-2}(K(i) !)
\end{aligned}
$$


Definitions for S and $\mathrm{K}(\mathrm{n} 0-1)$ :

$$
\begin{aligned}
& S=\sum_{i=0}^{i=n 0-2} K(i) \\
& K(n 0-1)=k+1-\left[\sum_{i=0}^{i=n 0-2}(K(i) *(n 0-i))\right]
\end{aligned}
$$

Condition $\mathrm{K}(\mathrm{n} 0-1) \geq 0$

This formula can be rewritten in the following expression:

$$
\begin{aligned}
& E(k)= \\
& \sum_{S=0}^{S=k}\left(\left(\sum_{i=0}^{i=n 0-2} K(i)+K(n 0-1)\right)\right) * \\
& \left.\left(\sum_{i=0}^{i=n 0-2} K(i)\right)\right) \\
& \prod_{i=0}^{i=n 0-2}(K(i) !)
\end{aligned}
$$

Definitions for S and $\mathrm{K}(\mathrm{n} 0-1)$ :

$$
\begin{aligned}
& S=\sum_{i=0}^{i=n 0-2} K(i) \\
& K(n 0-1)=k+1-\left[\sum_{i=0}^{i=n 0-2}(K(i) *(n 0-i))\right.
\end{aligned}
$$

condition $\mathrm{K}(\mathrm{n} 0-1) \geq 0$

Now we will develop the formula $\mathrm{E}(\mathrm{k})$ for the quintic equation $X^{5}=a X^{4}+b X^{3}+c X^{2}+d X+e$

The expression of $\mathrm{E}_{\mathrm{k}}$ developed for the qunitic formula.

$$
\begin{aligned}
E_{k}= & a^{k+1}+\left(\begin{array}{c}
k \\
1
\end{array}\right) a^{k-1} b+\left(\begin{array}{c}
k-1 \\
1
\end{array}\right) a^{k-2} c+\left(\begin{array}{c}
k-2 \\
1
\end{array}\right) a^{k-3} d+\left(\begin{array}{c}
k-3 \\
1
\end{array}\right) a^{k-4} e+\left(\begin{array}{c}
k-1 \\
2
\end{array}\right) a^{k-3} b^{2} \\
+ & 2\left(\begin{array}{c}
k-2 \\
2
\end{array}\right) a^{k-4} b c+2\left(\begin{array}{c}
k-3 \\
2
\end{array}\right) a^{k-5} b d+2\left(\begin{array}{c}
k-4 \\
2
\end{array}\right) a^{k-6} b e+2\left(\begin{array}{c}
k-4 \\
2
\end{array}\right) a^{k-6} c d+ \\
& 2\left(\begin{array}{c}
k-5 \\
2
\end{array}\right) a^{k-5} c^{2}+\left(\begin{array}{c}
k-5 \\
2
\end{array}\right) a^{k-7} d^{2}+\left(\begin{array}{c}
k-7 \\
2
\end{array}\right) a^{k-9} e^{2}+\left(\begin{array}{c}
k-2 \\
3
\end{array}\right) a^{k-5} b^{3}+3\left(\begin{array}{c}
k-3 \\
3
\end{array}\right) a^{k-6} b^{2} c+ \\
& 3\left(\begin{array}{c}
k-4 \\
3
\end{array}\right) a^{k-7} b c^{2}+\left(\begin{array}{c}
k-5 \\
3
\end{array}\right) a^{k-8} c^{3}+3\left(\begin{array}{c}
k-4 \\
3
\end{array}\right) a^{k-7} b^{2} d \\
& 3\left(\begin{array}{c}
k-6 \\
3
\end{array}\right) a^{k-9} b d^{2}+\left(\begin{array}{c}
k-8 \\
3
\end{array}\right) a^{k-11} d^{3}+ \\
& 3\left(\begin{array}{c}
k-5 \\
3
\end{array}\right) a^{k-8} b^{2} e+3\left(\begin{array}{c}
k-8 \\
3
\end{array}\right) a^{k-11} b c^{2}+ \\
& 6\left(\begin{array}{c}
k-5 \\
3
\end{array}\right) a^{k-8} b c d+12\left(\begin{array}{c}
k-7 \\
4
\end{array}\right) a^{k-11} b^{2} c e+ \\
& 12\left(\begin{array}{c}
k-8 \\
4
\end{array}\right) a^{k-12} b c^{2} e+12\left(\begin{array}{c}
k-10 \\
4
\end{array}\right) a^{k-14} b c e^{2}+ \\
& 12\left(\begin{array}{c}
k-10 \\
4
\end{array}\right) a^{k-14} c^{2} d e+24\left(\begin{array}{c}
k-11 \\
4
\end{array}\right) a^{k-15} c d^{2} e+ \\
& 12\left(\begin{array}{c}
k-12 \\
4
\end{array}\right) a^{k-16} c d e^{2}+24\left(\begin{array}{c}
k-9 \\
4
\end{array}\right) a^{k-13} b c d e+ \\
& 3\left(\begin{array}{c}
k-6 \\
3
\end{array}\right) a^{k-9} c^{2} d+3\left(\begin{array}{c}
k-7 \\
3
\end{array}\right) a^{k-10} c d^{2}+c\left(\begin{array}{c}
k-7 \\
3
\end{array}\right) a^{k-10} b d e+ \\
& 4\left(\begin{array}{c}
k-8 \\
4
\end{array}\right) a^{k-12} c^{3} d+6\left(\begin{array}{c}
k-9 \\
4
\end{array}\right) a^{k-13} c^{2} d^{2}+ \\
& 4\left(\begin{array}{c}
k-10 \\
4
\end{array}\right) a^{k-14} c d^{3}+4\left(\begin{array}{c}
k-9 \\
4
\end{array}\right) a^{k-13} c^{2} e+ \\
& 6\left(\begin{array}{c}
k-11 \\
4
\end{array}\right) a^{k-15} c^{2} e^{2}+4\left(\begin{array}{c}
k-13 \\
4
\end{array}\right) a^{k-17} d^{2} e^{2}+ \\
& 4\left(\begin{array}{c}
k-14 \\
4
\end{array}\right) a^{k-18} d e^{3}+6\left(\begin{array}{c}
k-5 \\
4
\end{array}\right) a^{k-9} b^{2} c^{2}+4\left(\begin{array}{c}
k-6 \\
4
\end{array}\right) a^{k-10} b c^{3}+
\end{aligned}
$$

$\left(\begin{array}{l}k-7 \\ 4\end{array}\right) a^{k-11} c^{4}+4\left(\begin{array}{c}k-5 \\ 4\end{array}\right) a^{k-9} b^{3} d+6\left(\begin{array}{l}k-7 \\ 4\end{array}\right) a^{k-11} b^{2} d^{2}+$

$4\left(\begin{array}{c}k-9 \\ 4\end{array}\right) a^{k-13} b d^{3}+\left(\begin{array}{c}k-11 \\ 4\end{array}\right) a^{k-15} d^{4}+4\left(\begin{array}{c}k-6 \\ 4\end{array}\right) a^{k-10} b^{3} c$

$+6\left(\begin{array}{c}k-9 \\ 4\end{array}\right) a^{k-13} b^{2} e^{2}+4\left(\begin{array}{c}k-12 \\ 4\end{array}\right) a^{k-16} b c^{3}+\left(\begin{array}{l}k-15 \\ 4\end{array}\right) a^{k-9} e^{4}+$

$\left(\begin{array}{l}k-5 \\ 6\end{array}\right) a^{k-11} b^{6}+6\left(\begin{array}{l}k-6 \\ 6\end{array}\right) a^{k-12} b^{5} c+15\left(\begin{array}{l}k-7 \\ 6\end{array}\right) a^{k-13} b^{4} c^{2}$

$20\left(\begin{array}{c}k-8 \\ 6\end{array}\right) a^{k-14} b^{3} c^{3}+15\left(\begin{array}{c}k-9 \\ 6\end{array}\right) a^{k-15} b^{2} c^{4}+$

$6\left(\begin{array}{c}k-10 \\ 6\end{array}\right) a^{k-16} b c^{5}+\left(\begin{array}{c}k-11 \\ 6\end{array}\right) a^{k-17} c^{6}+6\left(\begin{array}{c}k-7 \\ 6\end{array}\right) a^{k-13} b^{5} d+$

$30\left(\begin{array}{c}k-8 \\ 6\end{array}\right) a^{k-14} b^{4} c d+60\left(\begin{array}{c}k-9 \\ 6\end{array}\right) a^{k-15} b^{3} c^{2} d+$

$60\left(\begin{array}{c}k-10 \\ 6\end{array}\right) a^{k-16} b^{2} c^{3} d+30\left(\begin{array}{l}k-11 \\ 6\end{array}\right) a^{k-17} b c^{4} d+$

$6\left(\begin{array}{l}k-12 \\ 6\end{array}\right) a^{k-18} c^{5} d+6\left(\begin{array}{l}k-8 \\ 6\end{array}\right) a^{k-14} b^{5} c+30\left(\begin{array}{l}k-9 \\ 6\end{array}\right) a^{k-15} b^{4} c e+$

$60\left(\begin{array}{c}k-10 \\ 6\end{array}\right) a^{k-16} b^{2} c^{3} d+60\left(\begin{array}{c}k-11 \\ 6\end{array}\right) a^{k-17} b^{2} c^{3} e+30\left(\begin{array}{c}k-12 \\ 6\end{array}\right) a^{k-18} b c^{4} e+$

$6\left(\begin{array}{c}k-13 \\ 6\end{array}\right) a^{k-19} c^{5} e+30\left(\begin{array}{c}k-9 \\ 5\end{array}\right) a^{k-14} b^{2} c d^{2}+30\left(\begin{array}{c}k-10 \\ 5\end{array}\right) a^{k-15} b c^{2} d^{2}+$

$10\left(\begin{array}{c}k-11 \\ 5\end{array}\right) a^{k-16} c^{3} d^{2}+20\left(\begin{array}{c}k-9 \\ 5\end{array}\right) a^{k-17} c^{3} d e+60\left(\begin{array}{c}k-10 \\ 5\end{array}\right) a^{k-15} b^{2} c d e+$

$60\left(\begin{array}{l}k-11 \\ 5\end{array}\right) a^{k-16} b c^{2} d e+20\left(\begin{array}{l}k-12 \\ 5\end{array}\right) a^{k-17} c^{3} d e+10\left(\begin{array}{l}k-10 \\ 5\end{array}\right) b^{3} e^{2}+$

$30\left(\begin{array}{c}k-11 \\ 5\end{array}\right) a^{k-16} b^{2} c e^{2}+30\left(\begin{array}{c}k-12 \\ 5\end{array}\right) a^{k-17} b c^{2} e^{2}+15\left(\begin{array}{c}k-9 \\ 6\end{array}\right) a^{k-15} b^{4} d^{2}$

$+60\left(\begin{array}{c}k-10 \\ 6\end{array}\right) a^{k-16} b^{3} c d^{2}+90\left(\begin{array}{c}k-11 \\ 6\end{array}\right) a^{k-17} b^{2} c^{2} d^{2}+60\left(\begin{array}{c}k-12 \\ 6\end{array}\right) a^{k-18} b c^{3} d^{2}+$ $15\left(\begin{array}{c}k-13 \\ 6\end{array}\right) a^{k-19} c^{4} d^{2}+30\left(\begin{array}{c}k-10 \\ 6\end{array}\right) a^{k-16} b^{4} d e$

$120\left(\begin{array}{c}k-11 \\ 6\end{array}\right) a^{k-17} b^{3} c d e+100\left(\begin{array}{c}k-12 \\ 6\end{array}\right) a^{k-18} b^{2} c^{2} d e+$

$120\left(\begin{array}{c}k-13 \\ 6\end{array}\right) a^{k-19} b c^{3} d e+30\left(\begin{array}{c}k-14 \\ 6\end{array}\right) a^{k-20} c^{4} d e+5\left(\begin{array}{c}k-17 \\ 5\end{array}\right) a^{k-22} c e^{4}+$

$\left(\begin{array}{l}k-14 \\ 5\end{array}\right) a^{k-19} d^{5}+5\left(\begin{array}{l}k-15 \\ 5\end{array}\right) a^{k-20} d^{4} e+10\left(\begin{array}{l}k-16 \\ 5\end{array}\right) a^{k-21} d^{3} e^{2}+$

$10\left(\begin{array}{c}k-17 \\ 5\end{array}\right) a^{k-22} d^{2} e^{3}+5\left(\begin{array}{c}k-18 \\ 5\end{array}\right) a^{k-23} d e^{4}+\left(\begin{array}{c}k-19 \\ 5\end{array}\right) a^{k-24} e^{5}+$

$20\left(\begin{array}{c}k-16 \\ 5\end{array}\right) a^{k-21} c d e^{3}+10\left(\begin{array}{c}k-10 \\ 5\end{array}\right) a^{k-15} b^{2} d^{3}+6\left(\begin{array}{c}k-6 \\ 3\end{array}\right) a^{k-9} b c e+$

$3\left(\begin{array}{c}k-7 \\ 3\end{array}\right) a^{k-10} c^{2} e+3\left(\begin{array}{c}k-9 \\ 3\end{array}\right) a^{k-12} c e^{2}+6\left(\begin{array}{c}k-8 \\ 3\end{array}\right) a^{k-11} c d e+$

$3\left(\begin{array}{c}k-9 \\ 3\end{array}\right) a^{k-12} d^{2} e+3\left(\begin{array}{c}k-10 \\ 3\end{array}\right) a^{k-13} d e^{2}+\left(\begin{array}{c}k-11 \\ 3\end{array}\right) a^{k-14} e^{3}+$

$\left(\begin{array}{c}k-3 \\ 4\end{array}\right) a^{k-7} b^{4}+4\left(\begin{array}{c}k-4 \\ 4\end{array}\right) a^{k-8} b^{3} c+10\left(\begin{array}{c}k-13 \\ 5\end{array}\right) a^{k-18} b^{2} e^{3}+$

$20\left(\begin{array}{c}k-14 \\ 5\end{array}\right) a^{k-19} b c e^{3}+10\left(\begin{array}{c}k-15 \\ 5\end{array}\right) a^{k-20} c^{2} e^{3}+5\left(\begin{array}{c}k-12 \\ 5\end{array}\right) a^{k-17} b d^{4}+$

$5\left(\begin{array}{l}k-13 \\ 5\end{array}\right) a^{k-18} b^{2} e^{2}+20\left(\begin{array}{c}k-13 \\ 5\end{array}\right) a^{k-18} b d^{3} e+5\left(\begin{array}{l}k-14 \\ 5\end{array}\right) a^{k-19} c d^{3} e+$

$30\left(\begin{array}{l}k-14 \\ 5\end{array}\right) a^{k-19} b d^{2} e^{2}+20\left(\begin{array}{c}k-15 \\ 5\end{array}\right) a^{k-20} b d e^{3}+5\left(\begin{array}{c}k-16 \\ 5\end{array}\right) a^{k-21} b e^{4}+$

$10\left(\begin{array}{c}k-13 \\ 5\end{array}\right) a^{k-18} c^{3} e^{2}+20\left(\begin{array}{c}k-11 \\ 5\end{array}\right) a^{k-16} b c d^{3}+10\left(\begin{array}{c}k-12 \\ 5\end{array}\right) a^{k-17} c^{2} d^{3}+$ 


$$
\begin{aligned}
& 30\left(\begin{array}{c}
k-11 \\
5
\end{array}\right) a^{k-16} b^{2} d^{2} e+60\left(\begin{array}{c}
k-12 \\
5
\end{array}\right) a^{k-17} b c d^{2} e+30\left(\begin{array}{c}
k-13 \\
5
\end{array}\right) a^{k-18} c^{2} d^{2} e+ \\
& 30\left(\begin{array}{c}
k-12 \\
5
\end{array}\right) a^{k-17} b^{2} d e^{2}+60\left(\begin{array}{c}
k-13 \\
5
\end{array}\right) a^{k-18} b c d e^{2}+30\left(\begin{array}{c}
k-14 \\
5
\end{array}\right) a^{k-19} c^{2} d e^{2}+ \\
& 30\left(\begin{array}{c}
k-19 \\
6
\end{array}\right) a^{k-25} b d e^{4}+30\left(\begin{array}{c}
k-20 \\
6
\end{array}\right) a^{k-26} c d e^{4}+6\left(\begin{array}{c}
k-20 \\
6
\end{array}\right) a^{k-26} b e^{5}+ \\
& 6\left(\begin{array}{c}
k-21 \\
6
\end{array}\right) a^{k-27} c e^{5}+6\left(\begin{array}{c}
k-18 \\
6
\end{array}\right) a^{k-27} d^{2} e^{4}+15\left(\begin{array}{c}
k-19 \\
6
\end{array}\right) a^{k-25} d^{4} e^{2}+ \\
& 20\left(\begin{array}{c}
k-20 \\
6
\end{array}\right) a^{k-26} d^{3} c^{3}+15\left(\begin{array}{c}
k-21 \\
6
\end{array}\right) a^{k-27} d^{2} e^{4}+6\left(\begin{array}{c}
k-22 \\
6
\end{array}\right) a^{k-28} d e^{5}+ \\
& \left(\begin{array}{c}
k-23 \\
6
\end{array}\right) a^{k-29} e^{6}+60\left(\begin{array}{c}
k-16 \\
6
\end{array}\right) a^{k-22} c^{3} d e^{2}+20\left(\begin{array}{c}
k-14 \\
6
\end{array}\right) a^{k-20} b^{3} e^{3}+ \\
& 60\left(\begin{array}{c}
k-15 \\
6
\end{array}\right) a^{k-24} b^{2} c e^{3}+60\left(\begin{array}{c}
k-16 \\
6
\end{array}\right) a^{k-22} b c^{2} e^{3}+20\left(\begin{array}{c}
k-17 \\
6
\end{array}\right) a^{k-23} c^{3} e^{3}+ \\
& 15\left(\begin{array}{c}
k-13 \\
6
\end{array}\right) a^{k-19} b^{2} d^{4}+30\left(\begin{array}{c}
k-14 \\
6
\end{array}\right) a^{k-20} b c d^{4}+15\left(\begin{array}{c}
k-15 \\
6
\end{array}\right) a^{k-21} c^{2} d^{4}+ \\
& 60\left(\begin{array}{c}
k-14 \\
6
\end{array}\right) a^{k-20} b^{2} d^{3} e+120\left(\begin{array}{c}
k-15 \\
6
\end{array}\right) a^{k-21} b c d^{3} e+ \\
& 60\left(\begin{array}{c}
k-12 \\
6
\end{array}\right) a^{k-18} b^{3} d^{2} e+100\left(\begin{array}{c}
k-13 \\
6
\end{array}\right) a^{k-19} b^{2} c d^{2} e+100\left(\begin{array}{c}
k-14 \\
6
\end{array}\right) a^{k-20} b c^{2} d^{2} e+ \\
& 60\left(\begin{array}{c}
k-15 \\
6
\end{array}\right) a^{k-21} c^{3} d^{2} e+60\left(\begin{array}{c}
k-13 \\
6
\end{array}\right) a^{k-19} b^{3} d e^{2}+100\left(\begin{array}{c}
k-14 \\
6
\end{array}\right) a^{k-20} b^{2} c d e^{2}+ \\
& 100\left(\begin{array}{c}
k-15 \\
6
\end{array}\right) a^{k-21} b c^{2} d e^{2}+15\left(\begin{array}{c}
k-11 \\
6
\end{array}\right) a^{k-17} b^{4} e^{2}+60\left(\begin{array}{c}
k-12 \\
6
\end{array}\right) a^{k-18} b^{3} c e^{2}+ \\
& 90\left(\begin{array}{c}
k-13 \\
6
\end{array}\right) a^{k-19} b^{2} c^{2} e^{2}+60\left(\begin{array}{c}
k-14 \\
6
\end{array}\right) a^{k-20} b c^{3} e^{2}+15\left(\begin{array}{c}
k-15 \\
6
\end{array}\right) a^{k-21} c^{4} e^{2}+ \\
& 20\left(\begin{array}{c}
k-11 \\
6
\end{array}\right) a^{k-17} b^{3} d^{3}+60\left(\begin{array}{l}
k-12 \\
6
\end{array}\right) a^{k-18} b^{2} c d^{3}+60\left(\begin{array}{c}
k-13 \\
6
\end{array}\right) a^{k-19} b c^{2} d^{3}+ \\
& 20\left(\begin{array}{c}
k-14 \\
6
\end{array}\right) a^{k-20} c^{3} d^{3}+60\left(\begin{array}{c}
k-16 \\
6
\end{array}\right) a^{k-22} c^{2} d^{3} e+90\left(\begin{array}{c}
k-15 \\
6
\end{array}, a^{k-21} b^{2} d^{2} e^{2}+\right. \\
& \left.100\left(\begin{array}{c}
k-16 \\
6
\end{array}\right) a^{k-22} b c d^{2} e^{2}+90\left(\begin{array}{c}
k-17 \\
6
\end{array}\right) a^{k-23} c^{2} d^{2} e^{2}+60_{6}^{k-16}\right) a^{k-22} c^{2} d e+ \\
& 120\left(\begin{array}{c}
k-17 \\
6
\end{array}\right) a^{k-23} b c d e^{3}+60\left(\begin{array}{c}
k-18 \\
6
\end{array}\right) a^{k-24} c^{2} d e^{3}+15\left(\begin{array}{c}
k-23 \\
6
\end{array}, a^{k-23} c^{2} e^{4}+\right. \\
& 30\left(\begin{array}{c}
k-18 \\
6
\end{array}\right) a^{k-24} b c e^{4}+15\left(\begin{array}{c}
k-19 \\
6
\end{array}\right) a^{k-25} c^{2} e^{4}+\left(\begin{array}{c}
k-17 \\
6
\end{array}\right) a^{k-23} d^{6}+ \\
& 6\left(\begin{array}{c}
k-15 \\
6
\end{array}\right) a^{k-21} b d^{5}+6\left(\begin{array}{c}
k-16 \\
6
\end{array}\right) a^{k-22} c d^{5}+30\left(\begin{array}{c}
k-16 \\
6
\end{array}\right) a^{k-22} b d^{4} e+ \\
& 30\left(\begin{array}{c}
k-17 \\
6
\end{array}\right) a^{k-23} c d^{4} e+60\left(\begin{array}{c}
k-17 \\
6
\end{array}\right) a^{k-23} b d^{3} e^{2}+60\left(\begin{array}{c}
k-18 \\
6
\end{array}\right) a^{k-24} c d^{3} e^{2}+ \\
& 60\left(\begin{array}{c}
k-15 \\
6
\end{array}\right) a^{k-24} b d^{2} e^{3}+60\left(\begin{array}{c}
k-19 \\
6
\end{array}\right) a^{k-25} c d^{2} e^{3}+20\left(\begin{array}{c}
k-9 \\
5
\end{array}\right) a^{k-14} b c^{3} d \\
& 5\left(\begin{array}{c}
k-10 \\
6
\end{array}\right) a^{k-15} c^{4} d+5\left(\begin{array}{c}
k-7 \\
5
\end{array}\right) a^{k-12} b^{4} e+20\left(\begin{array}{c}
k-8 \\
5
\end{array}\right) a^{k-13} b^{3} c e+ \\
& 30\left(\begin{array}{c}
k-9 \\
5
\end{array}\right) a^{k-14} b^{2} c^{2} e+20\left(\begin{array}{c}
k-10 \\
5
\end{array}\right) a^{k-15} b c^{3} e+5\left(\begin{array}{c}
k-11 \\
5
\end{array}\right) a^{k-16} c^{4} e+ \\
& 10\left(\begin{array}{c}
k-8 \\
5
\end{array}\right) a^{k-13} b^{3} d^{2}+\left(\begin{array}{c}
k-4 \\
5
\end{array}\right) a^{k-9} b^{5}+5\left(\begin{array}{c}
k-5 \\
5
\end{array}\right) a^{k-10} b^{4} c+ \\
& 10\left(\begin{array}{c}
k-6 \\
5
\end{array}\right) a^{k-11} b^{3} c^{2}+10\left(\begin{array}{c}
k-7 \\
5
\end{array}\right) a^{k-12} b^{2} c^{3}+5\left(\begin{array}{c}
k-8 \\
5
\end{array}\right) a^{k-13} b c^{4}+ \\
& \left(\begin{array}{c}
k-9 \\
5
\end{array}\right) a^{k-14} c^{5}+5\left(\begin{array}{c}
k-6 \\
5
\end{array}\right) a^{k-11} b^{4} d+20\left(\begin{array}{l}
k-7 \\
4
\end{array}\right) a^{k-12} b^{3} c d+ \\
& 12\left(\begin{array}{c}
k-7 \\
4
\end{array}\right) a^{k-11} b c^{2} d+12\left(\begin{array}{c}
k-8 \\
4
\end{array}\right) a^{k-12} b^{2} c^{3}+5\left(\begin{array}{c}
k-8 \\
4
\end{array}\right) a^{k-12} b^{2} d e+ \\
& 12\left(\begin{array}{c}
k-10 \\
4
\end{array}\right) a^{k-14} b d^{2} e+12\left(\begin{array}{c}
k-11 \\
4
\end{array}\right) a^{k-15} b d e^{2}+\left(\begin{array}{c}
k-6 \\
7
\end{array}\right) a^{k-13} b^{7}+ \\
& \left(\begin{array}{l}
k-7 \\
8
\end{array}\right) a^{k-15} b^{8}+7\left(\begin{array}{c}
k-7 \\
7
\end{array}\right) a^{k-14} b^{6} c+21\left(\begin{array}{l}
k-8 \\
7
\end{array}\right) a^{k-15} b^{5} c^{2}+7\left(\begin{array}{c}
k-8 \\
7
\end{array}\right) a^{k-15} b^{6} d
\end{aligned}
$$

Let's express the root of a quantic equation by using the expression of $\mathrm{E}_{\mathrm{k}}$ developed for the quinitic formula $\left(\mathrm{n}_{0}=5\right)$

\section{$X^{5}=3 X^{4}+4 X^{3}+2 X^{2}+X+1$.}

The first thing we need to determine is the minimum number of interaction needed to calculate the solution of the equation. This number is called $\mathrm{K}_{1}=\mathrm{K} 0+\mathrm{n} 0$ following the derivatives given to determine $\mathrm{Kc}$

We illustrate how to calculate $\mathrm{K}_{0}, \mathrm{~K}_{0}$ is the minimum common factor between

$1 \times 3,2 \times 4,3 \times 2,4 \times 1,5 \times 1$.
Where on the right side of each product is represented the coefficient of the quinitic equation and on the left side of each product is represented the indices starting from 1 .

So we need to identify the different pair of prime factors contained in the product between the five products.

$[1 \times 3] \times[2 \times 4] \times[3 \times 2] \times[4 \times 1] \times[5 \times 1]$

At next we need to express this product in terms of prime factors

$[1 \times 3] \times[2 \times 2 \times 2] \times[3 \times 2] \times[2 \times 2 \times 1] \times[5 \times 1]$.

The next thing we need to do is to divide this product by each pair of prime factors Let's do this for each pair of prime factors

$[1 \times 3] \times[2 \times 2 \times 2] \times[3 \times 2] \times[2 \times 2 \times 1] \times[5 \times 1] / 2 \times 2 \times 2 \times 2 \times 2 \times 3 \times 3=5$.

At next we need to determine the equivalent of this result in terms of modulo $\mathrm{n} 0$ when $\mathrm{n} 0$ is the degree of the equation since the equation is a quintic equation so $\mathrm{n} 0=5$.

So 5 modulo $5=5[5]=0$

So $\mathrm{k} 0=0$ and $\mathrm{k} 1=\mathrm{k} 0+\mathrm{n} 00+5=5$

So $\mathrm{K} 1=5$

So $\mathrm{K} 1=5$ interacts will give at least one digit after the decimal point of the equation.

For $\mathrm{m}$ digits after the decimal point So:

$$
K=(K \mathrm{o}+n 0)[1+2 \ldots . . m]=(K \mathrm{o}+n 0) * \mathrm{~m}(m+1) / 2
$$

This number $\mathrm{k}$ is the maximum number of the interactions needed to calculate the solutions until $\mathrm{m}$ digits after the decimal point.

So here $k=5 m *(m+1) / 2$

For $m=1 \quad K=5 * 1 *(1+1) / 2=5$

In the decimal system $\mathrm{K}+10=5+10=15$ interactions is the maximum number of the interactions for which $\mathrm{m}=1$ digits after the decimal point is also common to the subsequent terms of the series $G(\mathrm{~K}+1)=\frac{E(K+1)}{E(K)}$ which means that

$\mathrm{L}($ with $\mathrm{m}=1$ digit $)=\mathrm{G}(15)=\mathrm{G}(16)$

Infact checking the result with a calculator will show that $G(15)=\frac{E(15)}{E(14)}$ gives 9 digits after the decimal point of the solution x.

So $\mathrm{X}$ (with 9 digits after the decimal point) $=\left[\operatorname{int}\left(\mathrm{G}(15)^{\star} 10^{9}\right)\right] / 10^{9}$

Where int is the integers function Now we need to calculate $\mathrm{E}(15)$ and $\mathrm{E}(14)$ using the expression of $\mathrm{E}(\mathrm{k})$ developed for the quantic formula we need to remember that only positive powers are allowed in the expression.

We calculate $\mathrm{E}(14)$ So $\mathrm{k}=14, \mathrm{a}=3, \mathrm{~b}=4, \mathrm{c}=2, \mathrm{~d}=1, \mathrm{e}=1$ 


$$
E_{14}=3^{14+1}+\left(\begin{array}{l}
14 \\
1
\end{array}\right) \times 3^{14-1} \times\left(\begin{array}{c}
14-1 \\
1
\end{array}\right) \times 3^{14-2} \times 2+
$$

$\left(\begin{array}{l}14-2 \\ 1\end{array}\right) \times 3^{14-3} \times 1+\left(\begin{array}{l}14-3 \\ 1\end{array}\right) \times 3^{14-4} \times 1+\left(\begin{array}{c}14-1 \\ 2\end{array}\right) \times 3^{14-3} \times 4^{2}+$

$2\left(\begin{array}{c}14-2 \\ 2\end{array}\right) \times 3^{14-4} \times 4 \times 2+2\left(\begin{array}{c}14-3 \\ 2\end{array}\right) \times 3^{14-5} \times 4 \times 1+$

$\left(\begin{array}{c}14-7 \\ 2\end{array}\right) \times 3^{14-9} \times 1^{2}+2\left(\begin{array}{c}14-4 \\ 2\end{array}\right) \times 3^{14-6} \times 4 \times 1+$

$2\left(\begin{array}{c}14-4 \\ 2\end{array}\right) \times 3^{14-6} \times 2 \times 1+2\left(\begin{array}{c}14-5 \\ 2\end{array}\right) \times 3^{14-7} \times 2 \times 1+$

$2\left(\begin{array}{c}14-6 \\ 2\end{array}\right) \times 3^{14-8} \times 1 \times 1+\left(\begin{array}{c}14-3 \\ 2\end{array}\right) \times 3^{14-5} \times 2^{2}+$

$\left(\begin{array}{c}14-5 \\ 2\end{array}\right) \times 3^{14-7} \times 1^{2}+12\left(\begin{array}{c}14-6 \\ 4\end{array}\right) \times 3^{14-10} \times 4^{2} \times 2 \times 1+$

$12 \times\left(\begin{array}{c}14-7 \\ 4\end{array}\right) \times 3^{14-11} \times 4 \times 2^{2} \times 1+12\left(\begin{array}{c}14-8 \\ 4\end{array}\right) \times 3^{14-12} \times 4 \times 2 \times 1^{2}+$

$12\left(\begin{array}{c}14-10 \\ 4\end{array}\right) \times 3^{14-14} \times 4 \times 2 \times 1^{2}+12\left(\begin{array}{c}14-10 \\ 4\end{array}\right) \times 3^{14-14} \times 2^{2} \times 1 \times 1+$

$24\left(\begin{array}{c}14-9 \\ 4\end{array}\right) \times 3^{14-13} \times 4 \times 2 \times 1 \times 1+\left(\begin{array}{c}14-5 \\ 6\end{array}\right) \times 3^{14-11} \times 4^{6}+$

$\left(\begin{array}{c}14-2 \\ 3\end{array}\right) \times 3^{14-5} \times 4^{3} \times 2^{2}+\left(\begin{array}{c}14-5 \\ 3\end{array}\right) \times 3^{14-8} \times 2^{3}+$

$3\left(\begin{array}{c}14-4 \\ 3\end{array}\right) \times 3^{14-7} \times 4^{2} \times 1+3\left(\begin{array}{c}14-6 \\ 3\end{array}\right) \times 3^{14-9} \times 4 \times 1^{2}+$

$\left(\begin{array}{c}14-8 \\ 3\end{array}\right) \times 3^{14-11} \times 1^{3}+3\left(\begin{array}{c}14-5 \\ 3\end{array}\right) \times 3^{14-8} \times 4^{2} \times 1+$

$3\left(\begin{array}{c}14-8 \\ 3\end{array}\right) \times 3^{14-11} \times 4 \times 1^{2}+6\left(\begin{array}{c}14-5 \\ 3\end{array}\right) \times 3^{14-8} \times 4 \times 2 \times 1+$

$6\left(\begin{array}{c}14-5 \\ 4\end{array}\right) \times 3^{14-9} \times 4^{2} \times 2^{2}+4\left(\begin{array}{c}14-6 \\ 4\end{array}\right) \times 3^{14-10} \times 4 \times 2^{3}+$

$\left(\begin{array}{c}14-7 \\ 4\end{array}\right) \times 3^{14-11} \times 4^{2} \times 1^{2}+4\left(\begin{array}{c}14-5 \\ 4\end{array}\right) \times 3^{14-9} \times 4^{3} \times 1+$

$6\left(\begin{array}{c}14-7 \\ 4\end{array}\right) \times 3^{14-11} \times 4^{2} \times 1^{2}+4\left(\begin{array}{c}14-9 \\ 4\end{array}\right) \times 3^{14-13} \times 4 \times 1^{3}+$

$4\left(\begin{array}{c}14-6 \\ 4\end{array}\right) \times 3^{14-10} \times 4^{3} \times 1+6\left(\begin{array}{c}14-9 \\ 4\end{array}\right) \times 3^{14-13} \times 4^{2} \times 1^{2}+$

$3\left(\begin{array}{c}14-6 \\ 3\end{array}\right) \times 3^{14-9} \times 2^{2} \times 1+3\left(\begin{array}{c}14-7 \\ 3\end{array}\right) \times 3^{14-10} \times 2 \times 1^{2}+$

$6\left(\begin{array}{c}14-7 \\ 3^{14}\end{array}\right) \times 3^{14-10} \times 4 \times 1 \times 1+4\left(\begin{array}{c}14-8 \\ 4\end{array}\right) \times 3^{14-12} \times 2^{3} \times 1+$

$6\left(\begin{array}{c}14-9 \\ 4\end{array}\right) \times 3^{14-13} \times 2^{2} \times 1^{2}+4\left(\begin{array}{c}14-10 \\ 4\end{array}\right) \times 3^{14-14} \times 2 \times 1^{3}$

$4\left(\begin{array}{c}14-9 \\ 4\end{array}\right) \times 3^{14-13} \times 2^{3} \times 1+6\left(\begin{array}{c}14-6 \\ 3\end{array}\right) \times 3^{14-9} \times 4 \times 2 \times 1+$

$3\left(\begin{array}{c}14-7 \\ 3^{4}\end{array}\right) \times 3^{14-10} \times 2^{2} \times 1+3\left(\begin{array}{c}14-9 \\ 3\end{array}\right) \times 3^{14-12} \times 2 \times 1^{2}+$

$6\left(\begin{array}{c}14-8 \\ 3\end{array}\right) \times 3^{14-11} \times 2 \times 1 \times 1+3\left(\begin{array}{c}14-9 \\ 3\end{array}\right) \times 3^{14-14} \times 1^{3}+$

$\left(\begin{array}{c}14-3 \\ 4\end{array}\right) \times 3^{14-7} \times 4^{4}+4\left(\begin{array}{c}14-4 \\ 4\end{array}\right) \times 3^{14-8} \times 4^{3} \times 2-$

$\left(\begin{array}{c}14-6 \\ 7\end{array}\right) \times 3^{14-13} \times 4^{7}+7\left(\begin{array}{c}14-7 \\ 7\end{array}\right) \times 3^{14-14} \times 4^{6} \times 2+$

$30\left(\begin{array}{c}14-9 \\ 5\end{array}\right) \times 3^{14-14} \times 4^{2} \times 2 \times 1^{2}+20\left(\begin{array}{c}14-9 \\ 5\end{array}\right) \times 3^{14-14} \times 4^{3} \times 1 \times 1+$

$6\left(\begin{array}{c}14-6 \\ 4\end{array}\right) \times 3^{14-12} \times 4^{5} \times 2^{3}+6\left(\begin{array}{c}14-7 \\ 6\end{array}\right) \times 3^{14-13} \times 4^{5} \times 2^{2}+$

$20\left(\begin{array}{c}14-8 \\ 6\end{array}\right) \times 3^{14-14} \times 4^{3} \times 2^{3}+6\left(\begin{array}{c}14-7 \\ 6\end{array}\right) \times 3^{14-13} \times 4^{5} \times 1+$

$30\left(\begin{array}{c}14-8 \\ 6\end{array}\right) \times 3^{14-14} \times 4^{4} \times 2 \times 1+6\left(\begin{array}{c}14-8 \\ 6\end{array}\right) \times 3^{14-14} \times 4^{5} \times 1+$

$\left(\begin{array}{c}14-4 \\ 5\end{array}\right) \times 3^{14-9} \times 4^{5}+5\left(\begin{array}{c}14-5 \\ 6\end{array}\right) \times 3^{14-10} \times 4^{4} \times 2+$

$10\left(\begin{array}{c}14-6 \\ 5\end{array}\right) \times 3^{14-11} \times 4^{3} \times 2^{2}+10\left(\begin{array}{c}14-7 \\ 5\end{array}\right) \times 3^{14-12} \times 4^{2} \times 2^{3}+$

$5\left(\begin{array}{c}14-8 \\ 5\end{array}\right) \times 3^{14-13} \times 4 \times 2^{4}+\left(\begin{array}{c}14-9 \\ 5\end{array}\right) \times 3^{14-14} \times 2^{5}+$

$5\left(\begin{array}{c}14-6 \\ 5\end{array}\right) \times 3^{14-11} \times 4^{4} \times 1+20\left(\begin{array}{c}14-7 \\ 5\end{array}\right) \times 3^{14-12} \times 4^{3} \times 2 \times 1+$

$30\left(\begin{array}{c}14-8 \\ 5\end{array}\right) \times 3^{14-13} \times 4^{2} \times 2^{2} \times 1+20\left(\begin{array}{c}14-9 \\ 5\end{array}\right) \times 3^{14-14} \times 4 \times 2^{3} \times 1+$

$30\left(\begin{array}{c}14-9 \\ 5\end{array}\right) \times 3^{14-14} \times 4^{2} \times 2^{2} \times 1+10\left(\begin{array}{c}14-8 \\ 5\end{array}\right) \times 3^{14-13} \times 4^{3} \times 1^{2}$

\section{$\mathrm{E}(14)=1231215656$.}

At next we calculate $\mathrm{E}_{15}, \mathrm{k}=15 \mathrm{a}=3, \mathrm{~b}=4, \mathrm{c}=2, \mathrm{~d}=1, \mathrm{e}=1$

$$
E_{15}=3^{15+1}+\left(\begin{array}{l}
15 \\
1
\end{array}\right) \times 3^{15-1} \times 4+\left(\begin{array}{c}
15-1 \\
1
\end{array}\right) \times 3^{15-2} \times 2-
$$

$\left(\begin{array}{l}15-2 \\ 1\end{array}\right) \times 3^{15-3} \times 1+\left(\begin{array}{c}15-3 \\ 1\end{array}\right) \times 3^{15-4} \times 1+\left(\begin{array}{c}15-1 \\ 2\end{array}\right) \times 3^{15-3} \times 4^{2}+$

$2\left(\begin{array}{c}15-2 \\ 2\end{array}\right) \times 3^{15-4} \times 4 \times 2+2\left(\begin{array}{c}15-3 \\ 2\end{array}\right) \times 3^{15-5} \times 4 \times 1+$

$\left(\begin{array}{c}15-7 \\ 2\end{array}\right) \times 3^{15-9} \times 1^{2}+2\left(\begin{array}{c}15-4 \\ 2\end{array}\right) \times 3^{15-6} \times 4 \times 1+$

$2\left(\begin{array}{c}15-4 \\ 2\end{array}\right) \times 3^{15-6} \times 2 \times 1+2\left(\begin{array}{c}15-5 \\ 2\end{array}\right) \times 3^{15-7} \times 2 \times 1+$

$2\left(\begin{array}{c}15-6 \\ 2\end{array}\right) \times 3^{15-8} \times 1 \times 1+\left(\begin{array}{c}15-3 \\ 2\end{array}\right) \times 3^{15-5} \times 2^{2}+$

$\left(\begin{array}{c}15-5 \\ 2\end{array}\right) \times 3^{15-7} \times 1^{2}+12\left(\begin{array}{c}15-6 \\ 4\end{array}\right) \times 3^{15-10} \times 4^{2} \times 2 \times 1+$

$12\left(\begin{array}{c}15-7 \\ 4\end{array}\right) \times 3^{15-11} \times 4 \times 2^{2} \times 1+12\left(\begin{array}{c}15-8 \\ 4\end{array}\right) \times 3^{15-12} \times 4 \times 2 \times 1^{2}+$

$12\left(\begin{array}{c}15-8 \\ 4\end{array}\right) \times 3^{15-12} \times 4^{2} \times 1 \times 1+12\left(\begin{array}{c}15-10 \\ 4\end{array}\right) \times 3^{15-14} \times 4 \times 1^{2} \times 1+$

$12\left(\begin{array}{c}15-11 \\ 4\end{array}\right) \times 3^{15-15} \times 4 \times 1 \times 1^{2}+12\left(\begin{array}{c}15-7 \\ 4\end{array}\right) \times 3^{15-11} \times 4^{2} \times 2 \times 1+$

$12\left(\begin{array}{c}15-8 \\ 4\end{array}\right) \times 3^{15-12} \times 4 \times 2^{2} \times 1+12\left(\begin{array}{c}15-10 \\ 4\end{array}\right) \times 3^{15-14} \times 4 \times 2 \times 1^{2}+$

$12\left(\begin{array}{c}15-10 \\ 4\end{array}\right) \times 3^{15-14} \times 2^{2} \times 1 \times 1+12\left(\begin{array}{c}15-11 \\ 4\end{array}\right) \times 3^{15-15} \times 2 \times 1^{2} \times 1+$

$24\left(\begin{array}{c}15-9 \\ 4\end{array}\right) \times 3^{15-13} \times 4 \times 2 \times 1 \times 1+\left(\begin{array}{c}15-5 \\ 6\end{array}\right) \times 3^{15-11} \times 4^{6}+$

$\left(\begin{array}{c}15-2 \\ 3\end{array}\right) \times 3^{15-15} \times 4 \times 2 \times 1 \times 1+\left(\begin{array}{c}15-5 \\ 6\end{array}\right) \times 3^{15-11} \times 4^{6}+$

$\left(\begin{array}{c}15-2 \\ 3\end{array}\right) \times 3^{15-15} \times 4^{3}+3\left(\begin{array}{c}15-3 \\ 3\end{array}\right) \times 3^{15-6} \times 4^{2} \times 2+$

$3\left(\begin{array}{c}15-4 \\ 3\end{array}\right) \times 3^{15-7} \times 4 \times 2^{2}+\left(\begin{array}{c}15-5 \\ 3\end{array}\right) \times 3^{15-8} \times 2^{3}+$

$3\left(\begin{array}{c}15-4 \\ 3\end{array}\right) \times 3^{15-7} \times 4^{2} \times 1+3\left(\begin{array}{c}15-6 \\ 3\end{array}\right) \times 3^{15-9} \times 4 \times 1^{2}+$

$\left(\begin{array}{c}15-8 \\ 3\end{array}\right) \times 3^{15-11} \times 1^{3}+3\left(\begin{array}{c}15-5 \\ 3\end{array}\right) \times 3^{15-8} \times 4^{2} \times 1+$

$3\left(\begin{array}{c}15-8 \\ 3\end{array}\right) \times 3^{15-11} \times 4 \times 1^{2}+6\left(\begin{array}{c}15-5 \\ 3\end{array}\right) \times 3^{15-8} \times 4 \times 2 \times 1+$

$6\left(\begin{array}{c}15-5 \\ 4\end{array}\right) \times 3^{15-9} \times 4^{2} \times 2^{2}+4\left(\begin{array}{c}15-6 \\ 4\end{array}\right) \times 3^{15-10} \times 4 \times 2^{3}+$

$\left(\begin{array}{c}15-7 \\ 4\end{array}\right) \times 3^{15-11} \times 2^{4}+4\left(\begin{array}{c}15-5 \\ 4\end{array}\right) \times 3^{15-9} \times 4^{3} \times 1+$

$6\left(\begin{array}{c}15-7 \\ 4\end{array}\right) \times 3^{15-11} \times 4^{2} \times 1^{2}+4\left(\begin{array}{c}15-9 \\ 4\end{array}\right) \times 3^{15-13} \times 4 \times 1^{3}+$

$\left(\begin{array}{c}15-11 \\ 4\end{array}\right) \times 3^{15-15} \times 1^{4}+4\left(\begin{array}{c}15-6 \\ 4\end{array}\right) \times 3^{15-10} \times 4^{3} \times 1+$

$6\left(\begin{array}{c}15-9 \\ 4\end{array}\right) \times 3^{15-13} \times 4^{2} \times 1^{2}+3\left(\begin{array}{c}15-6 \\ 3\end{array}\right) \times 3^{15-9} \times 2^{2} \times 1+$

$3\left(\begin{array}{c}15-7 \\ 3^{2}\end{array}\right) \times 3^{15-10} \times 2 \times 1^{6}+6\left(\begin{array}{c}15-7 \\ 3\end{array}\right) \times 3^{15-10} \times 4 \times 1 \times 1+$

$4\left(\begin{array}{c}15-8 \\ 4\end{array}\right) \times 3^{15-12} \times 2^{3} \times 1++6\left(\begin{array}{c}15-9 \\ 4\end{array}\right) \times 3^{15-13} \times 2^{3} \times 1+$

$4\left(\begin{array}{c}15-10 \\ 4\end{array}\right) \times 3^{15-14} \times 2 \times 1^{3}+4\left(\begin{array}{c}15-9 \\ 4\end{array}\right) \times 3^{15-13} \times 2^{3} \times 1+$

$6\left(\begin{array}{c}15-11 \\ 4\end{array}\right) \times 3^{15-15} \times 2^{2} \times 1^{2}+6\left(\begin{array}{c}15-6 \\ 3\end{array}\right) \times 3^{15-9} \times 4 \times 2 \times 1+$

$3\left(\begin{array}{c}15-7 \\ 4\end{array}\right) \times 3^{15-10} \times 2^{2} \times 1+3\left(\begin{array}{c}15-9 \\ 3\end{array}\right) \times 3^{15-12} \times 2 \times 1^{2}+$

$6\left(\begin{array}{c}15-8 \\ 3\end{array}\right) \times 3^{15-11} \times 2 \times 1 \times 1+3\left(\begin{array}{c}15-9 \\ 3\end{array}\right) \times 3^{15-12} \times 1^{2} \times 1+$

$3\left(\begin{array}{c}15-10 \\ 3\end{array}\right) \times 3^{15-13} \times 1 \times 1^{2}+\left(\begin{array}{c}15-11 \\ 3\end{array}\right) \times 3^{15-14} \times 1^{3}+$

$\left(\begin{array}{c}15-3 \\ 4\end{array}\right) \times 3^{15-7} \times 4^{4}+4\left(\begin{array}{c}15-4 \\ 4\end{array}\right) \times 3^{15-8} \times 4^{3} \times 2+$

$10\left(\begin{array}{c}15-10 \\ 5\end{array}\right) \times 3^{15-15} \times 4^{2} \times 1^{3}+15\left(\begin{array}{c}15-9 \\ 6\end{array}\right) \times 3^{15-15} \times 4^{4} \times 1^{2}+$

$\left(\begin{array}{c}15-6 \\ 7\end{array}\right) \times 3^{15-13} \times 4^{7}+\left(\begin{array}{c}15-7 \\ 8\end{array}\right) \times 3^{15-15} \times 4^{8}+$

$7\left(\begin{array}{c}15-7 \\ 7\end{array}\right) \times 3^{15-14} \times 4^{6} \times 2+21\left(\begin{array}{c}15-8 \\ 7\end{array}\right) \times 3^{15-15} \times 4^{5} \times 2^{2}+$

$7\left(\begin{array}{c}15-8 \\ 7\end{array}\right) \times 3^{15-15} \times 4^{6} \times 1+30\left(\begin{array}{c}15-9 \\ 5\end{array}\right) \times 3^{15-14} \times 4^{2} \times 2 \times 1^{2}+$

$30\left(\begin{array}{c}15-10 \\ 5\end{array}\right) \times 3^{15-15} \times 4 \times 2^{2} \times 1^{2}+20\left(\begin{array}{c}15-9 \\ 5\end{array}\right) \times 3^{15-14} \times 4^{3} \times 1 \times 1+$

$60\left(\begin{array}{c}15-10 \\ 5\end{array}\right) \times 3^{15-15} \times 4^{2} \times 2 \times 1 \times 1+10\left(\begin{array}{c}15-10 \\ 5\end{array}\right) \times 3^{15-15} \times 4^{3} \times 1^{2}+$

$6\left(\begin{array}{c}15-6 \\ 6\end{array}\right) \times 3^{15-12} \times 4^{5} \times 2+15\left(\begin{array}{c}15-7 \\ 6\end{array}\right) \times 3^{15-13} \times 4^{4} \times 1^{2}+$

$20\left(\begin{array}{c}15-8 \\ 6\end{array}\right) \times 3^{15-14} \times 4^{3} \times 2^{3}+15\left(\begin{array}{c}15-9 \\ 6\end{array}\right) \times 3^{15-15} \times 4^{2} \times 2^{4}+$

$6\left(\begin{array}{c}15-7 \\ 6\end{array}\right) \times 3^{15-13} \times 4^{5} \times 1+30\left(\begin{array}{c}15-8 \\ 6\end{array}\right) \times 3^{15-14} \times 4^{4} \times 2 \times 1+$ 


$$
\begin{aligned}
& 60\left(\begin{array}{c}
15-9 \\
6
\end{array}\right) \times 3^{15-15} \times 4^{3} \times 2^{2} \times 1+6\left(\begin{array}{c}
15-8 \\
6
\end{array}\right) \times 3^{15-14} \times 4^{5} \times 1+ \\
& 30\left(\begin{array}{c}
15-9 \\
6
\end{array}\right) \times 3^{15-15} \times 4^{4} \times 2 \times 1+\left(\begin{array}{c}
15-4 \\
5
\end{array}\right) \times 3^{15-9} \times 4^{5}+ \\
& 5\left(\begin{array}{l}
15-5 \\
5
\end{array}\right) \times 3^{15-10} \times 4^{2} \times 2^{3}+5\left(\begin{array}{c}
15-6 \\
5
\end{array}\right) \times 3^{15-11} \times 4^{3} \times 2^{2}+ \\
& 10\left(\begin{array}{c}
15-7 \\
5
\end{array}\right) \times 3^{15-12} \times 4^{2} \times 2^{3}+5\left(\begin{array}{c}
15-8 \\
5
\end{array}\right) \times 3^{15-13} \times 4 \times 2^{4}+ \\
&\left(\begin{array}{c}
15-9 \\
5
\end{array}\right) \times 3^{15-14} \times 2^{5}+5\left(\begin{array}{c}
15-6 \\
5
\end{array}\right) \times 3^{15-11} \times 4^{4} \times 1+ \\
& 20\left(\begin{array}{c}
15-7 \\
5
\end{array}\right) \times 3^{15-12} \times 4^{3} \times 2 \times 1+30\left(\begin{array}{c}
15-8 \\
5
\end{array}\right) \times 3^{15-13} \times 4^{2} \times 2^{2} \times 1+ \\
& 20\left(\begin{array}{c}
15-9 \\
5
\end{array}\right) \times 3^{15-14} \times 4 \times 2^{3} \times 1+5\left(\begin{array}{c}
15-10 \\
5
\end{array}\right) \times 3^{15-15} \times 2^{4} \times 1+ \\
& 5\left(\begin{array}{c}
15-7 \\
5
\end{array}\right) \times 3^{15-12} \times 4^{4} \times 1+20\left(\begin{array}{c}
15-8 \\
5
\end{array}\right) \times 3^{15-13} \times 4^{3} \times 2 \times 1+ \\
& 30\left(\begin{array}{c}
15-9 \\
5
\end{array}\right) \times 3^{15-14} \times 4^{2} \times 2^{2} \times 1+20\left(\begin{array}{l}
15-10 \\
5
\end{array}\right) \times 3^{15-15} \times 4 \times 2^{3} \times 1+ \\
& 10\left(\begin{array}{l}
15-8 \\
5
\end{array}\right) \times 3^{15-13} \times 4^{3} \times 1^{2}
\end{aligned}
$$

\section{$\mathrm{E}(15)=5059866125$.}

So let's calculate the solution of the quantic equation:

$$
\underset{\substack{\text { withthitits } \\ \text { afier the decimal } \\ \text { point }}}{X}=\frac{\operatorname{int}\left(G_{5} \times 10^{9}\right)}{10^{9}}=\frac{\operatorname{int}\left(\frac{E_{15}}{E_{14}} \times 10^{9}\right)}{10^{9}}=\frac{\operatorname{int}\left(\frac{5059866125}{1231215656} \times 10^{9}\right)}{10^{9}}=4,109650572
$$

So $\mathrm{X}=4.109650572$ is a solution of the quantic equation. $\mathrm{X}^{5}=3 \mathrm{X}^{4}+4 \mathrm{X}^{3}+2 \mathrm{X}^{2}+\mathrm{X}+1$.

This result can be checked by the Newton method or any other technique.

This How J determined the different power combinations in the $\mathrm{E}(\mathrm{K})$ formula for $\mathrm{K}=15$ and $\mathrm{K}=14$.

Let's denote $\mathrm{K} 0, \mathrm{~K} 1, \mathrm{~K} 2, \mathrm{~K} 3$ the respective process of b,c,d,e in the qunitic equation.

\section{$\mathrm{X}^{5}=\mathrm{a} X^{4}+b X^{3}+c X^{2}+d X+e$} $\mathrm{k} 3=0$.

For $\mathrm{k} 0+\mathrm{k} 1+\mathrm{k} 2+\mathrm{k} 3=0$ there is only one possibility $\mathrm{k} 0=0, \mathrm{k} 1=0, \mathrm{k} 2=0$,

For $\mathrm{k} 0+\mathrm{k} 1+\mathrm{k} 2+\mathrm{k} 3=1$ this is equivalent to $(\mathrm{k} 0+\mathrm{k} 1)+(\mathrm{k} 2+\mathrm{k} 3)=1$, so the possibilities for this are

$(\mathrm{k} 0+\mathrm{k} 1)=0,(\mathrm{k} 2+\mathrm{k} 3)=1$ or $(\mathrm{k} 0+\mathrm{k} 1)=1,(\mathrm{k} 2+\mathrm{k} 3)=0$

For $(\mathrm{k} 0+\mathrm{k} 1)=1$ the possibilities are $\mathrm{k} 0=0, \mathrm{k} 1=0$.

For $(\mathrm{k} 2+\mathrm{k} 3)=1$ the possibilities are $\mathrm{k} 2=0, \mathrm{k} 3=1$ or $\mathrm{k} 2=1, \mathrm{k} 3=0$.

For $(\mathrm{k} 0+\mathrm{k} 1)=1$ the possibilities are $\mathrm{k} 2=0, \mathrm{k} 1=1$ or $\mathrm{k} 0=1, \mathrm{k} 1=0$

For $(\mathrm{k} 2+\mathrm{k} 3)=0$ the possibilities are $\mathrm{k} 2=0, \mathrm{k} 3=0$

So for $\mathrm{k} 0+\mathrm{k} 1+\mathrm{k} 2+\mathrm{k} 3=1$ the possibilities are

$$
\left(\begin{array}{cccc}
K_{0} & K_{1} & K_{2} & K_{3} \\
0 & 0 & 0 & 1 \\
0 & 0 & 1 & 0 \\
0 & 1 & 0 & 0 \\
1 & 0 & 0 & 0
\end{array}\right)
$$
$\mathrm{d}^{\mathrm{k} 2} \mathrm{e}^{\mathrm{k} 3}$

This gives the followings different power combinations for $b^{k 0} c^{k 1}$

$$
\begin{aligned}
& b^{0} c^{0} d^{0} e=e \\
& b^{0} c^{0} d^{0}=d
\end{aligned}
$$

\begin{tabular}{|c|c|c|c|}
\hline KO + & k1 + & k2 + & $k 3=2$ \\
\hline 0 & 0 & 0 & 2 \\
\hline 0 & 0 & 1 & 1 \\
\hline 0 & 0 & 2 & 0 \\
\hline 0 & 1 & 0 & 1 \\
\hline 0 & 1 & 1 & 0 \\
\hline 1 & 0 & 0 & 1 \\
\hline 1 & 0 & 1 & 0 \\
\hline 0 & 2 & 0 & 0 \\
\hline 1 & 1 & 0 & 0 \\
\hline 2 & 0 & 0 & 0 \\
\hline KO + & k1 + & k2 + & $k 3=3$ \\
\hline 0 & 0 & 0 & 3 \\
\hline 0 & 0 & 1 & 2 \\
\hline 0 & 0 & 2 & 1 \\
\hline 0 & 0 & 3 & 0 \\
\hline 0 & 1 & 0 & 2 \\
\hline 1 & 0 & 0 & 2 \\
\hline 0 & 1 & 1 & 1 \\
\hline 1 & 0 & 1 & 1 \\
\hline 0 & 1 & 2 & 0 \\
\hline 1 & 0 & 2 & 0 \\
\hline 0 & 2 & 0 & 1 \\
\hline 1 & 1 & 0 & 1 \\
\hline 2 & 0 & 0 & 1 \\
\hline 0 & 2 & 1 & 0 \\
\hline 1 & 1 & 1 & 0 \\
\hline 2 & 0 & 1 & 0 \\
\hline 3 & 0 & 0 & 0 \\
\hline 2 & 1 & 0 & 0 \\
\hline 1 & 2 & 0 & 0 \\
\hline 0 & 3 & 0 & 0 \\
\hline KO + & k1 + & $k 2+$ & $k 3=4$ \\
\hline 2 & 0 & 2 & 0 \\
\hline 0 & 3 & 0 & 1 \\
\hline 1 & 2 & 0 & 1 \\
\hline 2 & 1 & 0 & 1 \\
\hline 3 & 0 & 0 & 1 \\
\hline 0 & 3 & 1 & 0 \\
\hline 1 & 2 & 1 & 0 \\
\hline 2 & 1 & 1 & 0 \\
\hline 3 & 0 & 1 & 0 \\
\hline 0 & 4 & 0 & 0 \\
\hline
\end{tabular}

$\mathrm{b}^{0} \mathrm{~cd}^{0} \mathrm{e}^{0}=\mathrm{c}$

$b c^{0} d^{0} e^{0}=b$

So we need to perform the same things

For $\mathrm{K}_{0}+\mathrm{K}_{1}+\mathrm{K}_{2}+\mathrm{K}_{3}=2, \mathrm{~K}_{0}+\mathrm{K}_{1}+\mathrm{K}_{2}+\mathrm{K}_{3}=3$

$\mathrm{K}_{0}+\mathrm{K}_{1}+\mathrm{K}_{2}+\mathrm{K}_{3}=4, \mathrm{~K}_{0}+\mathrm{K}_{1}+\mathrm{K}_{2}+\mathrm{K}_{3}=5, \mathrm{~K}_{0}+\mathrm{K}_{1}+\mathrm{K}_{2}+\mathrm{K}_{3}=6, \mathrm{~K}_{0}+\mathrm{K}_{1}+\mathrm{K}_{2}+\mathrm{K}_{3}=7$.

For the qunitic equation $\mathrm{X} 5=3 \mathrm{x}^{4}+4 \mathrm{x}^{3}+2 \mathrm{x}^{2}+\mathrm{x}+1$.

We needed $\mathrm{E}(15)$ and $\mathrm{E}(14)$

For $\mathrm{k} 0+\mathrm{k} 1+\mathrm{k} 2+\mathrm{k} 3>7$ the powers of $\mathrm{a}=3$ in $\mathrm{E}(15)$ will be negative and since the condition in the $\mathrm{E}(\mathrm{k})$ formula is $\mathrm{K}($ no- 1$) \geq 0$, which means that the powers of a is to be positive powers . So we need to step the summation $\mathrm{k} 0+\mathrm{k} 1+\mathrm{k} 2+\mathrm{k} 3$ at the sum equal to 7 to calculate $\mathrm{E}(15)$.

These are the tables for the sum $\mathrm{k} 0+\mathrm{k} 1+\mathrm{k} 2+\mathrm{k} 3$ (Table 1 ). 
Page 10 of 12

\begin{tabular}{|c|c|c|c|c|c|c|c|}
\hline 1 & 3 & 0 & 0 & 4 & 1 & 0 & 0 \\
\hline 2 & 2 & 0 & 0 & 5 & 0 & 0 & 0 \\
\hline 3 & 1 & 0 & 0 & KO + & k1 + & k2 + & $k 3=6$ \\
\hline 4 & 0 & 0 & 0 & 0 & 0 & 0 & 6 \\
\hline KO + & k1 + & k2+ & $k 3=5$ & 0 & 0 & 1 & 5 \\
\hline 0 & 0 & 0 & 5 & 0 & 0 & 2 & 4 \\
\hline 0 & 0 & 1 & 4 & 0 & 0 & 3 & 3 \\
\hline 0 & 0 & 2 & 3 & 0 & 0 & 4 & 2 \\
\hline 0 & 0 & 3 & 2 & 0 & 0 & 5 & 1 \\
\hline 0 & 0 & 4 & 1 & 0 & 0 & 6 & 0 \\
\hline 0 & 0 & 5 & 0 & 0 & 1 & 0 & 5 \\
\hline 0 & 1 & 0 & 4 & 1 & 0 & 0 & 5 \\
\hline 1 & 0 & 0 & 4 & 0 & 1 & 1 & 4 \\
\hline 0 & 1 & 1 & 3 & 1 & 0 & 1 & 4 \\
\hline 1 & 0 & 1 & 3 & 0 & 1 & 2 & 3 \\
\hline 0 & 1 & 2 & 2 & 1 & 0 & 2 & 3 \\
\hline 1 & 0 & 2 & 2 & 0 & 1 & 3 & 2 \\
\hline 0 & 1 & 3 & 1 & 1 & 0 & 3 & 2 \\
\hline 1 & 0 & 3 & 1 & 0 & 1 & 4 & 1 \\
\hline 0 & 1 & 4 & 0 & 1 & 0 & 4 & 1 \\
\hline 1 & 0 & 4 & 0 & 0 & 1 & 5 & 0 \\
\hline 0 & 2 & 0 & 3 & 1 & 0 & 5 & 0 \\
\hline 1 & 1 & 0 & 3 & 0 & 2 & 0 & 4 \\
\hline 2 & 0 & 0 & 3 & KO + & $k 1+$ & k2 + & $k 3=6$ \\
\hline 0 & 2 & 1 & 2 & 1 & 1 & 0 & 4 \\
\hline 1 & 1 & 1 & 2 & 2 & 0 & 0 & 4 \\
\hline $\mathrm{KO}+$ & k1 + & k2 + & $k 3=5$ & 0 & 2 & 1 & 3 \\
\hline 2 & 0 & 1 & 2 & 1 & 1 & 1 & 3 \\
\hline 0 & 2 & 2 & 1 & 2 & 0 & 1 & 3 \\
\hline 1 & 1 & 2 & 1 & 0 & 2 & 2 & 2 \\
\hline 2 & 0 & 2 & 1 & 1 & 1 & 2 & 2 \\
\hline 0 & 2 & 3 & 0 & 2 & 0 & 2 & 2 \\
\hline 1 & 1 & 3 & 0 & 0 & 2 & 3 & 1 \\
\hline 2 & 0 & 3 & 0 & 1 & 1 & 3 & 1 \\
\hline 0 & 3 & 0 & 2 & 2 & 0 & 3 & 1 \\
\hline 1 & 2 & 0 & 2 & 0 & 2 & 4 & 0 \\
\hline 2 & 1 & 0 & 2 & 1 & 1 & 4 & 0 \\
\hline 3 & 0 & 0 & 2 & 2 & 0 & 4 & 0 \\
\hline 0 & 3 & 1 & 1 & 0 & 3 & 0 & 3 \\
\hline 1 & 2 & 1 & 1 & 1 & 2 & 0 & 3 \\
\hline 2 & 1 & 1 & 1 & 2 & 1 & 0 & 3 \\
\hline 3 & 0 & 1 & 1 & 3 & 0 & 0 & 3 \\
\hline 0 & 3 & 2 & 0 & 0 & 3 & 1 & 2 \\
\hline 1 & 2 & 2 & 0 & 1 & 2 & 1 & 2 \\
\hline 2 & 1 & 2 & 0 & 2 & 1 & 1 & 2 \\
\hline 3 & 0 & 2 & 0 & KO + & k1+ & k2 + & $k 3=6$ \\
\hline 0 & 4 & 0 & 1 & 3 & 0 & 1 & 2 \\
\hline 1 & 3 & 0 & 1 & 0 & 3 & 2 & 1 \\
\hline $\mathrm{K} 0+$ & k1 + & k2+ & $k 3=5$ & 1 & 2 & 2 & 1 \\
\hline 2 & 2 & 0 & 1 & 2 & 1 & 2 & 1 \\
\hline 3 & 1 & 0 & 1 & 3 & 0 & 2 & 1 \\
\hline 4 & 0 & 0 & 1 & 0 & 3 & 3 & 0 \\
\hline 0 & 4 & 1 & 0 & 1 & 2 & 3 & 0 \\
\hline 1 & 3 & 1 & 0 & 2 & 1 & 3 & 0 \\
\hline 2 & 2 & 1 & 0 & 3 & 0 & 3 & 0 \\
\hline 3 & 1 & 1 & 0 & 0 & 4 & 0 & 2 \\
\hline 4 & 0 & 1 & 0 & 1 & 3 & 0 & 2 \\
\hline 0 & 5 & 0 & 0 & 2 & 2 & 0 & 2 \\
\hline 1 & 4 & 0 & 0 & 3 & 1 & 0 & 2 \\
\hline 2 & 3 & 0 & 0 & 4 & 0 & 0 & 1 \\
\hline 3 & 2 & 0 & 0 & 0 & 4 & 1 & 1 \\
\hline
\end{tabular}


Page 11 of 12

\begin{tabular}{|c|c|c|c|}
\hline 1 & 3 & 1 & 1 \\
\hline 2 & 2 & 1 & 1 \\
\hline 3 & 1 & 1 & 1 \\
\hline 4 & 0 & 1 & 1 \\
\hline 0 & 4 & 2 & 0 \\
\hline 1 & 3 & 2 & 0 \\
\hline $\mathrm{KO}+$ & k1 + & $k 2+$ & $k 3=6$ \\
\hline 2 & 2 & 2 & 0 \\
\hline 3 & 1 & 2 & 0 \\
\hline 4 & 0 & 2 & 0 \\
\hline 0 & 5 & 0 & 1 \\
\hline 1 & 4 & 0 & 1 \\
\hline 2 & 3 & 0 & 1 \\
\hline 3 & 2 & 0 & 1 \\
\hline 4 & 1 & 0 & 1 \\
\hline 5 & 0 & 0 & 1 \\
\hline 0 & 5 & 1 & 0 \\
\hline 1 & 4 & 1 & 0 \\
\hline 2 & 3 & 1 & 0 \\
\hline 3 & 2 & 1 & 0 \\
\hline 4 & 1 & 1 & 0 \\
\hline 5 & 0 & 1 & 0 \\
\hline 0 & 6 & 0 & 0 \\
\hline 1 & 5 & 0 & 0 \\
\hline 2 & 4 & 0 & 0 \\
\hline 3 & 3 & 0 & 0 \\
\hline 4 & 2 & 0 & 0 \\
\hline 5 & 1 & 0 & 0 \\
\hline $\mathrm{KO}+$ & $k 1+$ & $k 2+$ & $k 3=6$ \\
\hline 6 & 0 & 0 & 0 \\
\hline $\mathrm{KO}+$ & $k 1+$ & k2+ & $k 3=7$ \\
\hline 0 & 0 & 0 & 7 \\
\hline 0 & 0 & 1 & 6 \\
\hline 0 & 0 & 2 & 5 \\
\hline 0 & 0 & 3 & 4 \\
\hline 0 & 0 & 4 & 3 \\
\hline 0 & 0 & 5 & 2 \\
\hline 0 & 0 & 6 & 1 \\
\hline 0 & 0 & 7 & 0 \\
\hline 0 & 1 & 0 & 6 \\
\hline 1 & 0 & 0 & 6 \\
\hline 0 & 1 & 1 & 5 \\
\hline 1 & 0 & 1 & 5 \\
\hline 0 & 1 & 2 & 4 \\
\hline 1 & 0 & 2 & 4 \\
\hline 0 & 1 & 3 & 3 \\
\hline 1 & 0 & 3 & 3 \\
\hline 0 & 1 & 4 & 2 \\
\hline 1 & 0 & 4 & 2 \\
\hline 0 & 1 & 5 & 1 \\
\hline $\mathrm{KO}+$ & $k 1+$ & $k 2+$ & $k 3=7$ \\
\hline 1 & 0 & 5 & 1 \\
\hline 0 & 1 & 6 & 0 \\
\hline 1 & 0 & 6 & 0 \\
\hline 0 & 2 & 0 & 5 \\
\hline 1 & 1 & 0 & 5 \\
\hline 2 & 0 & 0 & 5 \\
\hline 0 & 2 & 1 & 4 \\
\hline 1 & 1 & 1 & 4 \\
\hline 2 & 0 & 1 & 4 \\
\hline 0 & 2 & 2 & 3 \\
\hline
\end{tabular}

\begin{tabular}{|c|c|c|c|}
\hline 1 & 1 & 2 & 3 \\
\hline 2 & 0 & 2 & 3 \\
\hline 0 & 2 & 3 & 2 \\
\hline 1 & 1 & 3 & 2 \\
\hline 2 & 0 & 3 & 2 \\
\hline 0 & 2 & 4 & 1 \\
\hline 1 & 1 & 4 & 1 \\
\hline 2 & 0 & 4 & 1 \\
\hline 0 & 2 & 5 & 0 \\
\hline 1 & 1 & 5 & 0 \\
\hline 2 & 0 & 5 & 0 \\
\hline KO + & $k 1+$ & k2 + & $k 3=7$ \\
\hline 0 & 3 & 0 & 4 \\
\hline 1 & 2 & 0 & 4 \\
\hline 2 & 1 & 0 & 4 \\
\hline 3 & 0 & 0 & 4 \\
\hline 0 & 3 & 1 & 3 \\
\hline 1 & 2 & 1 & 3 \\
\hline 2 & 1 & 1 & 3 \\
\hline 3 & 0 & 1 & 3 \\
\hline 0 & 3 & 2 & 2 \\
\hline 1 & 2 & 2 & 2 \\
\hline 2 & 1 & 2 & 2 \\
\hline 3 & 0 & 2 & 2 \\
\hline 0 & 3 & 3 & 1 \\
\hline 1 & 2 & 3 & 1 \\
\hline 2 & 1 & 3 & 1 \\
\hline 3 & 0 & 3 & 1 \\
\hline 0 & 3 & 4 & 0 \\
\hline 1 & 2 & 4 & 0 \\
\hline 2 & 1 & 4 & 0 \\
\hline 3 & 0 & 4 & 0 \\
\hline 0 & 4 & 0 & 3 \\
\hline Ko + & $k 1+$ & k2+ & $k 3=7$ \\
\hline 1 & 3 & 0 & 3 \\
\hline 2 & 2 & 0 & 3 \\
\hline 3 & 1 & 0 & 3 \\
\hline 4 & 0 & 0 & 3 \\
\hline 0 & 4 & 1 & 2 \\
\hline 1 & 3 & 1 & 2 \\
\hline 2 & 2 & 1 & 2 \\
\hline 3 & 1 & 1 & 2 \\
\hline 4 & 0 & 1 & 2 \\
\hline 0 & 4 & 2 & 1 \\
\hline 1 & 3 & 2 & 1 \\
\hline 2 & 2 & 2 & 1 \\
\hline 3 & 1 & 2 & 1 \\
\hline 4 & 0 & 2 & 1 \\
\hline 0 & 4 & 3 & 0 \\
\hline 1 & 3 & 3 & 0 \\
\hline 2 & 2 & 3 & 0 \\
\hline 3 & 1 & 3 & 0 \\
\hline 4 & 0 & 3 & 0 \\
\hline 0 & 5 & 0 & 2 \\
\hline 1 & 4 & 0 & 2 \\
\hline KO + & $k 1+$ & k2 + & $k 3=7$ \\
\hline 2 & 3 & 0 & 2 \\
\hline 3 & 2 & 0 & 2 \\
\hline 4 & 1 & 0 & 2 \\
\hline 5 & 0 & 0 & 2 \\
\hline 0 & 5 & 1 & 1 \\
\hline
\end{tabular}




\begin{tabular}{|c|c|c|c|}
\hline 1 & 4 & 1 & 1 \\
\hline 2 & 3 & 1 & 1 \\
\hline 3 & 2 & 1 & 1 \\
\hline 4 & 1 & 1 & 1 \\
\hline 5 & 0 & 1 & 1 \\
\hline 0 & 5 & 2 & 0 \\
\hline 1 & 4 & 2 & 0 \\
\hline 2 & 3 & 2 & 0 \\
\hline 3 & 2 & 2 & 0 \\
\hline 4 & 1 & 2 & 0 \\
\hline 5 & 0 & 2 & 0 \\
\hline 0 & 6 & 0 & 1 \\
\hline 1 & 5 & 0 & 1 \\
\hline 2 & 4 & 0 & 1 \\
\hline 3 & 3 & 0 & 1 \\
\hline 4 & 2 & 0 & 1 \\
\hline $\mathrm{KO}+$ & k1+ & k2+ & $k 3=7$ \\
\hline 5 & 1 & 0 & 1 \\
\hline 6 & 0 & 0 & 1 \\
\hline 0 & 6 & 1 & 0 \\
\hline 1 & 5 & 1 & 0 \\
\hline 2 & 4 & 1 & 0 \\
\hline 3 & 3 & 1 & 0 \\
\hline 4 & 2 & 1 & 0 \\
\hline 5 & 1 & 1 & 0 \\
\hline 6 & 0 & 1 & 0 \\
\hline 0 & 7 & 0 & 0 \\
\hline 1 & 6 & 0 & 0 \\
\hline 2 & 5 & 0 & 0 \\
\hline 3 & 4 & 0 & 0 \\
\hline 4 & 3 & 0 & 0 \\
\hline 5 & 2 & 0 & 0 \\
\hline 6 & 1 & 0 & 0 \\
\hline 7 & 0 & 0 & 0 \\
\hline
\end{tabular}

\section{References}

1. Coppersmith Don (2001) "Finding a small root of a univariate modular equation". International Conference on the Theory and Applications of Cryptographic Techniques, pp:155-165.

2. Faugère JC (1999) "A new efficient algorithm for computing Gröbner bases (F4). Journal of Pure and Applied Algebra 139: 61-88.

3. Allgower EL, Kurt Georg (1990) Numerical continuation methods. Springer Series in Computational Mathematics 13.

4. Courtois N, Klimov A, Patarin J, Shamir A (2000) Efficient Algorithms for Solving Over defined Systems of Multivariate Polynomial Equations. International Conference on the Theory and Applications of Cryptographic Techniques, pp: 392-407.

5. Gershon Elber, Myung-Soo Kim (2001) Geometric constraint solver using multivariate rational spline functions. In Proceedings of the sixth ACM Symposium on Solid Modelling and Applications, pp: 1-10.

6. Farin G (1990) Curves and surfaces for computer aided geometric design: A practical guide. Comp. science and sci. computing.

7. Patrikalakis MP, Maekawa T (2002) Shape Interrogation for Computer Aided Design and Manufacturing. Springer Verlag

8. Farouki RT, Goodman TNT (1996) On the optimal stability of the Bernstein basis. Mathematics of computation, 65: 1553-1566.

Table 1: The summation $\mathrm{k} 0+\mathrm{k} 1+\mathrm{k} 2+\mathrm{k} 3$ at the sum equal to 7 